\title{
On non-linear frequency chirping in connection with collisions
}

\author{
Christoph Slaby*, Axel Könies, Ralf Kleiber, and Henry Leyh \\ Max-Planck-Institut für Plasmaphysik, \\ Wendelsteinstraße 1, 17491 Greifswald, Germany
}

(Dated: December 10, 2018)

\begin{abstract}
The non-linear behaviour of toroidicity-induced Alfvén eigenmodes, destabilized by fast ions, is investigated in tokamak geometry and for a Wendelstein 7-X high-mirror equilibrium. Both cases show frequency chirping in the non-linear phase. The focus of this paper is on how particle collisions influence the non-linear dynamics and the associated frequency chirping. Pitch-angle scattering and fast-ion drag, which together are described by the fast-ion collision operator, are considered. We study the effect of a Krook operator, relaxing the distribution function to its unperturbed value, on the non-linear dynamics. The Krook operator leads to a periodic reappearance of the chirping. This is also observed in experiments in which a fast-particle source is usually present.

The simulations are carried out using the non-linear and fully three-dimensional CKAEUTERPE model. The model is perturbative in the sense that a fixed mode structure is used. Since such an investigation is undertaken for the first time for the stellarator Wendelstein 7-X, the tokamak case as well as analytical theory are used for comparison. The parameters of the fast-ion distribution function in Wendelstein 7-X are inspired by the 2018 experimental campaign which, for the first time, includes neutral beam injection to supply fast ions.
\end{abstract}

\footnotetext{
* E-mail address: christoph.slaby@ipp.mpg.de
} 


\section{INTRODUCTION}

In plasmas that are relevant for nuclear fusion, there typically exists a small population of fast ions that are much hotter than the bulk of the plasma. In today's fusion experiments such particles are created by neutral beam injection (NBI) or ion cyclotron resonance heating (ICRH). In future fusion reactors, alpha particles created by the deuterium-tritium fusion reaction will be present. In order to heat the plasma, the fast ions need to remain in the plasma long enough to thermalize.

However, in the process of slowing down, the fast ions may resonantly interact with Alfvénic perturbations $[15,34]$. This can lead to enhanced fast-ion transport and also to the ejection of fast ions from the confinement region, which implies energy losses $[11,37]$. In severe cases, damage to plasma-facing components is possible [10, 11].

It depends on the magnetic equilibrium as well as on the fast-ion distribution function which Alfvén eigenmode (AE) in particular is most easily destabilized by the fast particles. A good overview is given in Ref. [18]. Typically, so-called gap modes that reside in frequency gaps of the shear Alfvén spectrum are likely to be unstable, because they experience only small continuum damping.

Especially with regard to future fusion reactors, an understanding of AE-induced fastion transport and redistribution has to be developed. Since the modes responsible for the redistribution are excited by the energetic ions themselves, the problem is non-linear. Analytical theory is available in the form of the simplified Berk-Breizman paradigm [1-4] which shows that a variety of non-linear behaviours - ranging from explosive, chirping, and periodic solutions to a steady-state dynamics - are possible. The analytical theory is able to identify some key parameters that govern the non-linear dynamics: The relative strength of damping and drive as well as diffusion and drag (i.e. collisions) are found to be important. Also the presence of particle sources can change the non-linear dynamics.

Frequency chirping has also been investigated experimentally in stellarators and tokamaks. AEs chirping in frequency are seen in the TJ-II stellarator in discharges that combine NBI and electron cyclotron resonance heating [31,32]. A transition from a chirping state to a steady state is linked to variations of the magnetic configuration [31], i.e. equilibrium changes. The fast-ion transport induced by frequency-chirping AEs has been investigated in the ASDEX Upgrade tokamak [16]. Comprehensive non-linear simulations of chirping AEs, including equilibrium changes and collisions, have been carried out for JT-60U plasmas [6].

This paper reports on non-linear simulations of toroidicity-induced Alfvén eigenmodes (TAEs) in tokamak and stellarator plasmas. We choose the TAE modes destabilized by fast particles as an example, standing in for the class of all AEs. We focus on the frequency chirping of TAEs excited in realistic geometry. All cases chosen for this investigation show frequency chirping in the absence of collisions. We will then investigate the impact of various collision operators on the frequency chirping. This is the first time that such an investigation is undertaken for Wendelstein $7-X(W 7-X)$. This study is of practical relevance for $W 7-X$, since it is one step needed towards the development of a full fast-ion transport model, which is still lacking.

We use the CKA-EUTERPE code package [14] for our numerical simulations. The re- 
duced ideal-magnetohydrodynamic (MHD) code CKA $[13,21]$ calculates the eigenmode (frequency and mode structure). In a separate step, the global non-linear gyro-kinetic electromagnetic particle-in-cell (PIC) code EUTERPE [20, 24] is used to compute the motion of marker particles in the pre-calculated field and the power transfer of the fast particles to the mode, which determines the temporal evolution of the mode amplitude. This approach is applied to two different magnetic configurations: We start by investigating how collisions influence the chirping in tokamaks. As an example, the ITPA benchmark case $[22,23]$ is used. Secondly, chirping TAEs influenced by collisions are simulated in a Wendelstein 7-X high-mirror equilibrium.

Various collision operators are explored in the paper. We start by using a simple pitchangle scattering operator. The effect of pitch-angle scattering on the saturation amplitudes of TAEs in the ITPA tokamak and in W7-X was recently computed in Ref. [36]. The pitch-angle-scattering operator is, of course, just a first approximation of the full fast-ion collision operator, which also includes slowing-down drag. The effect of drag will be studied separately from the pitch-angle collisions. Finally, we will use a Krook term [5], emulating a particle source, to relax the distribution function towards its initial state. The Krook operator is linked to periodic, well separated chirping events that are routinely observed in experiments [16, 31, 32] and numerical simulations [25-27, 29].

Note that the effects of collisions on chirping have been studied extensively [3, 4, 2530] in one-dimensional models that are related to the original Berk-Breizman paradigm. Relatively little work has been invested to study non-linear frequency chirping in twodimensional (see e.g. Ref. [6] for simulation results on frequency chirping in JT-60U plasmas) or even three-dimensional devices. This is the reason why we start this investigation with the (relatively simple) ITPA benchmark case: We recover many features predicted by the Berk-Breizman theory, but now in a two-dimensional system. The magnetic geometry is still simple enough that this case can bridge the gap to the complex W7-X case, where it is investigated whether the fully three-dimensional system leads to differences compared with the analytical theory.

The paper is organized as follows: Sec. II elaborates on the existing theory and introduces our numerical tools. Sec. III describes both the tokamak and the W7-X case. The non-linear dynamics in the collisionless limit is, for comparison, given in this section. How these results change when collisions are taken into account is shown in Secs. IV and V. Finally, conclusions are drawn in Sec. VI.

\section{THEORY AND ALGORITHMS}

\section{A. The numerical model of CKA-EUTERPE}

The theory behind the model of CKA-EUTERPE has recently been described in Ref. [36]. Therefore, only the defining characteristics of the model will be repeated here.

The CKA-EUTERPE code package combines the reduced ideal MHD code CKA (Code for Kinetic Alfvén waves) with the global non-linear $\delta \mathrm{f}$ particle-in-cell code EUTERPE. Both are suited for fully three-dimensional geometries.

CKA is an eigenvalue code used to solve for the frequency $\omega$ of the mode as well as the mode structure (in terms of the electrostatic potential $\phi$ ). Note that for vanishing 
parallel electric field, the electrostatic potential and the parallel component of the vector potential, $A_{\|}$, are linked via

$$
A_{\|}=\frac{\mathrm{i}}{\omega} \mathbf{b} \cdot \nabla \phi .
$$

( $\mathbf{b}$ denotes a unit vector in the direction of the magnetic field, $\mathbf{B}=B \mathbf{b}$.) The Coulomb gauge is used and the perpendicular component of the vector potential (related to compression of the magnetic field) is neglected. No kinetic fast-ion effects are considered in CKA.

The mode frequency, electrostatic potential, and vector potential are passed to EUTERPE, which solves the gyro-kinetic equation

$$
\frac{\partial f_{s}}{\partial t}+\dot{\mathbf{R}} \cdot \nabla f_{s}+\dot{v}_{\|} \frac{\partial f_{s}}{\partial v_{\|}}+\dot{\mu} \frac{\partial f_{s}}{\partial \mu}=\mathcal{C}^{s \bar{s}}\left(f_{s}, f_{\bar{s}}\right)
$$

for the distribution function of a species $s$ including a collision operator $\mathcal{C}^{s \bar{s}}\left(f_{s}, f_{\bar{s}}\right)$. In the CKA-EUTERPE model only the fast ions are treated gyro-kinetically. Therefore the species index will be omitted hereafter. $\mu$ denotes the specific magnetic moment $\mu=$ $v_{\perp}^{2} /(2 B)$. EUTERPE as a PIC code solves the kinetic equation by following numerical marker particles that move along the characteristics of Eq. (2). We split the distribution function into two parts, $f=f^{(0)}+f^{(1)}$, where $f^{(0)}$ is a time-independent background and $f^{(1)}$ denotes a perturbation, respectively. CKA-EUTERPE uses the so-called $v_{\|}$formulation of gyro-kinetics. Hence, in the collisionless limit and with the non-linear terms retained the equations of motion for the particles (characteristics of Eq. (2)) are

$$
\begin{aligned}
\dot{\mathbf{R}}= & v_{\|} \mathbf{b}+\frac{m}{q}\left[\frac{\mu B+v_{\|}^{2}}{B B_{\|}^{\star}} \mathbf{b} \times \nabla B+\frac{v_{\|}^{2}}{B B_{\|}^{\star}}(\nabla \times \mathbf{B})_{\perp}\right] \\
& +\frac{v_{\|}}{B B_{\|}^{\star}}\left[\mathbf{b} \times \nabla B+(\nabla \times \mathbf{B})_{\perp}\right]\left\langle A_{\|}\right\rangle+\frac{1}{B_{\|}^{\star}} \mathbf{b} \times \nabla\left\langle\phi-v_{\|} A_{\|}\right\rangle \\
\dot{v}_{\|}= & -\mu \nabla B \cdot\left[\mathbf{b}+\frac{m}{q} \frac{v_{\|}}{B B_{\|}^{\star}}(\nabla \times \mathbf{B})_{\perp}\right] \\
& -\frac{v_{\|}}{B B_{\|}^{\star}}\left[\mathbf{b} \times \nabla B+(\nabla \times \mathbf{B})_{\perp}\right] \cdot \nabla\langle\phi\rangle \\
& -\frac{\mu}{B_{\|}^{\star}}\left[\mathbf{b} \times \nabla B \cdot \nabla\left\langle A_{\|}\right\rangle+\frac{1}{B} \nabla B \cdot(\nabla \times \mathbf{B})_{\perp}\left\langle A_{\|}\right\rangle\right] \\
\dot{\mu}= & 0
\end{aligned}
$$

with

$$
B_{\|}^{\star}=B+\left[\frac{m}{q} v_{\|}+\left\langle A_{\|}\right\rangle\right] \mathbf{b} \cdot \nabla \times \mathbf{b} .
$$

Here, $m$ and $q$ denote the particle mass and charge, respectively. The angular brackets, $\langle\ldots\rangle$, denote the gyro-average. Note that the equation for $\dot{v}_{\|}$does not contain a time derivative of $A_{\|}$as we have used the $E_{\|}=0$ constraint to replace this time derivative with the parallel gradient of $\phi$. 
We emphasize again that the mode structure is fixed throughout the calculation. Therefore, neither Poisson's equation nor Ampère's law have to be solved. We only need to consider the temporal evolution of the amplitudes (denoted by a circumflex below) of the electromagnetic potentials. The equations for the amplitudes are given as (We refer to Refs. [21,36] for details.)

$$
\begin{aligned}
\frac{\partial \hat{\phi}(t)}{\partial t} & =\mathrm{i} \omega\left(\hat{A}_{\|}-\hat{\phi}\right)+2\left(\gamma(t)-\gamma_{\mathrm{d}}\right) \hat{\phi} \\
\frac{\partial \hat{A}_{\|}(t)}{\partial t} & =\mathrm{i} \omega\left(\hat{\phi}-\hat{A}_{\|}\right),
\end{aligned}
$$

where $\gamma(t)=P /(2 W)$ is a time-dependent growth rate that is computed from the waveparticle power transfer

$$
P=-\int \mathrm{d} \Gamma B_{\|}^{\star}\left[\frac{m}{Z \mathrm{e} B} \mathbf{b} \times\left(v_{\|}^{2} \boldsymbol{\kappa}+\mu \nabla B\right) \cdot\left(\mathrm{Ze} \nabla_{\perp} \phi^{*}(\mathbf{r}, t) f^{(1)}\right)\right]
$$

and wave energy

$$
W=\int \mathrm{d}^{3} r \frac{\rho}{B^{2}}\left|\nabla_{\perp} \phi\right|^{2} .
$$

Here, $\rho$ is the mass density of the plasma, the particle charge is denoted by Ze, $\kappa$ is the curvature of the magnetic field, and $\mathrm{d} \Gamma$ denotes an infinitesimal phase-space element. The electrostatic potential is given by $\phi(\mathbf{r}, t)=\hat{\phi}(t) \phi_{0}(\mathbf{r}) \exp (\mathrm{i} \omega t)$, where $\phi_{0}$ denotes the MHD eigenfunction of the electrostatic potential calculated by CKA (similar for $A_{\|}$). In Eqs. (3) and (4), the real parts of the potentials are taken to compute the particle trajectories. $\gamma_{\mathrm{d}}$ is an externally specified damping rate. Note that while the mode varies on a short time scale, the amplitudes in Eqs. (7) and (8) evolve much more slowly. This fact has been used in the derivation of the amplitude equations. Thus the model is only applicable for chirping which satisfies $\delta \omega / \omega \ll 1$.

\section{B. Collision operators}

Throughout this paper various collision operators will be used and compared with each other. Since the focus is on TAEs excited by fast ions, we start with the general expression for a collision operator of test particles $a$ colliding with a Maxwellian background [19] of particles $b$

$$
\mathcal{C}^{a b}\left(f_{a}^{(1)}, f_{b}^{\mathrm{M}}\right)=v_{\mathrm{D}}^{a b} \mathcal{L}\left(f_{a}^{(1)}\right)+\frac{1}{v^{2}} \frac{\partial}{\partial v}\left[v^{3}\left(\frac{m_{a}}{m_{a}+m_{b}} v_{\mathrm{s}}^{a b} f_{a}^{(1)}+\frac{1}{2} v_{\|}^{a b} v \frac{\partial f_{a}^{(1)}}{\partial v}\right)\right] .
$$

The first term in this general collision operator describes pitch-angle scattering of species $a$ with species $b$

$$
\nu_{\mathrm{D}}^{a b} \mathcal{L}=\mathcal{C}_{\text {pitch }}^{a b}=\frac{\nu_{\mathrm{D}}^{a b}}{2} \frac{\partial}{\partial \xi}\left(1-\xi^{2}\right) \frac{\partial}{\partial \xi}
$$

$\xi=v_{\|} / v$ denotes the pitch-angle variable,

$$
v_{\mathrm{D}}^{a b}=v_{0}^{a b} \frac{\Phi\left(x_{b}\right)-G\left(x_{b}\right)}{x_{a}^{3}}
$$


is the deflection frequency of species $a$ colliding with species $b[19]$, and $x_{s}=v /\left(\sqrt{2} v_{\text {th,s }}\right)$ with $v_{\mathrm{th}, \mathrm{s}}=\sqrt{\mathrm{k}_{\mathrm{B}} T_{s} / m_{s}}$. $T$ denotes the temperature. $\Phi$ and $G$ are the error function and the Chandrasekhar function, respectively. With $n$ being the density, $Z$ the charge number, e the electron charge, $\epsilon_{0}$ the vacuum permittivity, and $\ln \Lambda$ the Coulomb logarithm, we will use [19]

$$
v_{0}^{a b}=\frac{n_{b} Z_{a}^{2} Z_{b}^{2} e^{4} \ln \Lambda}{4 \pi \epsilon_{0}^{2} m_{a}^{2} 2^{3 / 2} v_{\text {th,a }}^{3}},
$$

with the density and temperature evaluated at the particle position, to determine the collision frequencies from the profiles. We will call this the self-consistent approach. However, in some cases it is enlightening to modify $v_{0}^{a b}$ in order to enforce a different non-linear behaviour of the system. Cases where this approach is pursued are indicated below. Note that in a realistic setting, the fast particles do not just collide among themselves. For typical fusion plasmas, the collision frequency with the bulk is many orders of magnitude larger, i.e. $v_{0}^{\mathrm{ff}} \ll v_{0}^{\mathrm{fi}}$. This motivates our approach to treat $v_{0}=\sum_{\bar{s}} v_{0}^{\mathrm{fs}}$ as an 'effective' collision frequency that contains different physics, depending on the case and which species are considered collisional. The letters ' $i$ ', ' $\mathrm{e}^{\prime}$ ', and ' $\mathrm{f}$ ' are used to label ions, electrons, and fast ions, respectively.

The implementation of the pitch-angle collision operator into EUTERPE is described in detail in Refs. $[9,36]$.

Furthermore, going back to Eq. (11), $v_{\mathrm{s}}^{a b}$ is the slowing-down frequency defined as [19]

$$
v_{\mathrm{s}}^{a b}=v_{0}^{a b} \frac{2 T_{a}}{T_{b}}\left(1+\frac{m_{b}}{m_{a}}\right) \frac{G\left(x_{b}\right)}{x_{a}},
$$

and $v_{\|}^{a b}$ denotes the parallel velocity diffusion frequency [19]. To arrive at a fast-ion collision operator, we employ the following approximations:

1. The combination $a=\mathrm{f}, b=\mathrm{f}$ can be neglected due to the high temperature and small density of the fast particles.

2. Parallel velocity diffusion can be neglected in general, since its frequency is typically very small.

3. Pitch-angle scattering of the fast ions off electrons can be neglected due to the mass difference.

Under these circumstances the original operator can be significantly simplified to

$$
\begin{aligned}
\mathcal{C}_{\text {fast }}\left(f_{\mathrm{f}}^{(1)}\right) & =\sum_{\bar{s}=\mathrm{i}, \mathrm{e}} \mathcal{C}_{f \bar{s}}\left(f_{\mathrm{f}}^{(1)}, f_{\overline{\mathrm{s}}}^{\mathrm{M}}\right) \\
& \cong v_{\mathrm{D}}^{\mathrm{fi}} \mathcal{L}\left(f_{\mathrm{f}}^{(1)}\right)+\frac{1}{v^{2}} \frac{\partial}{\partial v}\left[v^{3} \frac{m_{\mathrm{f}}}{m_{\mathrm{f}}+m_{\mathrm{i}}} v_{\mathrm{s}}^{\mathrm{fi}} f_{\mathrm{f}}^{(1)}\right]+\frac{1}{v^{2}} \frac{\partial}{\partial v}\left[v^{3} \frac{m_{\mathrm{f}}}{m_{\mathrm{f}}+m_{\mathrm{e}}} v_{\mathrm{s}}^{\mathrm{fe}} f_{\mathrm{f}}^{(1)}\right] .
\end{aligned}
$$

The first term describes pitch-angle collisions between fast and bulk ions. The second and the third term describe the drag that the fast ions experience with the background ions and electrons, respectively. 
It is tempting to further simplify the operator by using the fact that in fusion plasmas the fast particles are faster than the bulk ions, but still slower than the electrons. Therefore, one often uses the large-argument expansion of the Chandrasekhar function in the second term of Eq. (17). Accordingly, the third term is treated using the small-argument expansion, so that the operator can finally be written in the well-known form

$$
\mathcal{C}_{\text {fast }}\left(f_{\mathrm{f}}^{(1)}\right) \approx v_{\mathrm{D}}^{\mathrm{fi}} \mathcal{L}\left(f_{\mathrm{f}}^{(1)}\right)+\frac{1}{v^{2} \tau_{\mathrm{s}}} \frac{\partial}{\partial v}\left[\left(v^{3}+v_{\mathrm{c}}^{3}\right) f_{\mathrm{f}}^{(1)}\right],
$$

where $\tau_{\mathrm{s}}$ and $v_{\mathrm{c}}$ denote the slowing-down time and the critical velocity (below which ion drag is stronger than electron drag), respectively:

$$
\begin{aligned}
\tau_{\mathrm{s}} & =\frac{3(2 \pi)^{3 / 2} \epsilon_{0}^{2} m_{\mathrm{f}} \mathrm{k}_{\mathrm{B}}^{3 / 2} T_{\mathrm{e}}^{3 / 2}}{Z_{\mathrm{f}}^{2} \mathrm{e}^{4} \sqrt{m_{\mathrm{e}}} n_{\mathrm{e}} \ln \Lambda} \\
v_{\mathrm{c}} & =\sqrt{2} v_{\text {th, }}\left(\frac{n_{\mathrm{i}} Z_{\mathrm{i}}^{2}}{n_{\mathrm{e}}} \frac{3 \sqrt{\pi} m_{\mathrm{e}}}{4 m_{\mathrm{i}}}\right)^{1 / 3} .
\end{aligned}
$$

However, the approximated operator given in Eq. (18) presents certain challenges in practical applications. The approximation is only valid as long as $v_{\text {th,e }} \gg v_{\text {th,f }} \gg v_{\text {th,i }}$ is fulfilled. In a PIC code, however, the particles are loaded with a velocity distribution where some 'fast' particles will have velocities comparable to the bulk ions making the classification as fast particles misleading. Especially for those slow particles, the effect of drag is greatly enhanced by the approximations. In a stellarator this can lead to a localization (trapping) of particles in regions of low magnetic field strength. This localization of particles with respect to the toroidal angle of the device conflicts with the parallelization scheme (domain decomposition) and can lead to a severe load imbalance. This is the reason why we do not use the simplified fast-ion collision operator often cited in the literature, but instead use the one given by Eq. (17).

Recall that this operator is composed of a pitch-angle part and a drag part. In the code, an operator splitting is employed. The pitch-angle part can be treated in the way described in Refs. $[9,36]$. The drag part, which contains only a first-order derivative with respect to velocity, can be incorporated into the regular equations of motion, i.e. the derivative $\partial / \partial v$ is translated into $\partial / \partial v_{\|}$and $\partial / \partial \mu$ derivatives that are added to Eqs. (4) and (5). One finds that the changes of $v_{\|}$and $\mu$ due to drag are

$$
\begin{aligned}
\dot{v}_{\|, \text {drag }} & =-2^{3 / 2} T_{\mathrm{f}} v_{\text {th, } \mathrm{f}}\left[\frac{v_{0}^{\mathrm{fi}}}{T_{\mathrm{i}}} G\left(x_{\mathrm{i}}\right)+\frac{v_{0}^{\mathrm{fe}}}{T_{\mathrm{e}}} G\left(x_{\mathrm{e}}\right)\right] \xi \\
\dot{\mu}_{\text {drag }} & =-2^{3 / 2} T_{\mathrm{f}} v_{\text {th }, \mathrm{f}}\left[\frac{v_{0}^{\mathrm{fi}}}{T_{\mathrm{i}}} G\left(x_{\mathrm{i}}\right)+\frac{v_{0}^{\mathrm{fe}}}{T_{\mathrm{e}}} G\left(x_{\mathrm{e}}\right)\right] \frac{v\left(1-\xi^{2}\right)}{B} .
\end{aligned}
$$

The last operator that will be used in this paper is a simple Krook operator [5]. The role of this operator is to relax the distribution function to its unperturbed state by damping the perturbation at rate $v_{\text {Krook }}$.

Since we use the so-called $v_{\|}$-formulation of gyro-kinetics, the Krook operator is defined as

$$
\mathcal{C}_{\text {Krook }}\left(f^{(1)}\right)=-v_{\text {Krook }} f^{(1)}=-v_{\text {Krook }}\left(f-f^{(0)}\right) .
$$



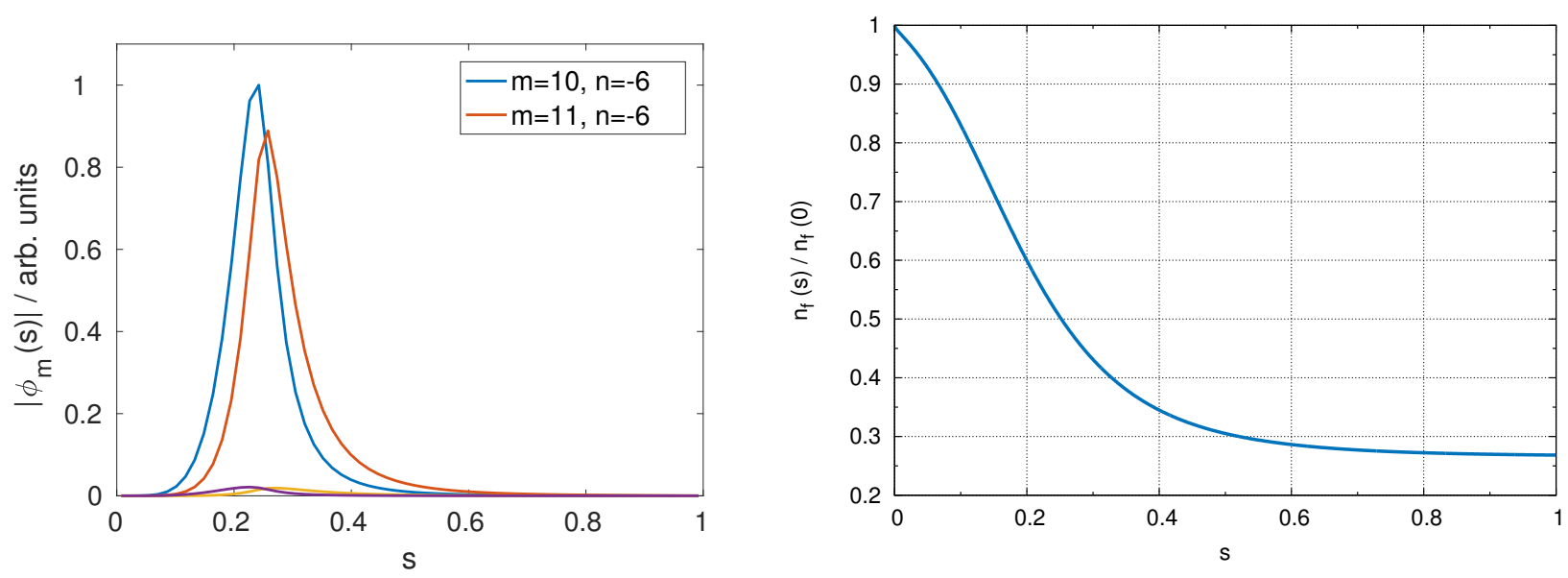

Figure 1: Mode structure (left) and normalized fast-ion density profile (right) for the ITPA case.

The motivation of using a Krook operator is as follows: Recall that in the onedimensional Berk-Breizman paradigm a gradient in velocity space is the source of the instability. This explains the very strong influence of pitch-angle collisions (acting in velocity space) within that model. In our case, the TAE is destabilized by a density gradient in real space. Accordingly, pitch-angle collisions will have a different effect on the non-linear dynamics in the present case. Therefore, we use a Krook operator to relax the distribution function to its initial state and thus to rebuild the gradients in real space. In this way the Krook operator can play a comparable role in our model as pitch-angle collisions do in the Berk-Breizman framework.

Throughout the paper, guiding-centre diffusion due to collisions is neglected.

\section{CASES AND COLLISIONLESS RESULTS}

In this section, we investigate the non-linear chirping behaviour of the ITPA tokamak case $[22,23]$ as well as that of a Wendelstein $7-X$ high-mirror case. The magnetic equilibrium and properties of the fast-particle distribution function are described briefly in this section. For later comparison, we will present the collisionless results here.

\section{A. Case description}

The ITPA tokamak case [22, 23] has been studied extensively in the past. The radial mode structure and the fast-ion density profile are shown in Fig. 1. The radial coordinate $s$ denotes the normalized toroidal flux. The background-plasma density and temperature profiles are flat with $n_{\mathrm{i}}=n_{\mathrm{e}}=2.0 \cdot 10^{19} \mathrm{~m}^{-3}$ and $T_{\mathrm{i}}=T_{\mathrm{e}}=1 \mathrm{keV}$, respectively. We take the usual TAE mode, but vary the fast-ion content in the plasma in order to change the linear growth rate $\gamma_{\mathrm{L}}=\gamma(t)+\gamma_{\mathrm{d}}$ and thus cause different non-linear scenarios. $\gamma(t)$ is the instantaneous growth rate as measured by the code. It is constant in the linear phase and drops when saturation is reached. Thus, $\gamma_{\mathrm{L}}$ is only meaningful in the linear phase and corresponds to the growth rate without damping. The on-axis 
Table I: Fast-ion densities and resulting linear growth rates for the tokamak cases.

\begin{tabular}{c|c|c}
\hline \hline case & Density $n_{\mathrm{f}}(0) / \mathrm{m}^{-3}$ & Linear growth rate $\gamma_{\mathrm{L}} / \mathrm{s}^{-1}$ \\
\hline tokamak case 1 & $7.24 \cdot 10^{16}$ & $1.25 \cdot 10^{4}$ \\
\hline tokamak case 2 & $2.51 \cdot 10^{17}$ & $4.05 \cdot 10^{4}$ \\
\hline tokamak case 3 & $5.03 \cdot 10^{17}$ & $7.53 \cdot 10^{4}$ \\
\hline \hline
\end{tabular}

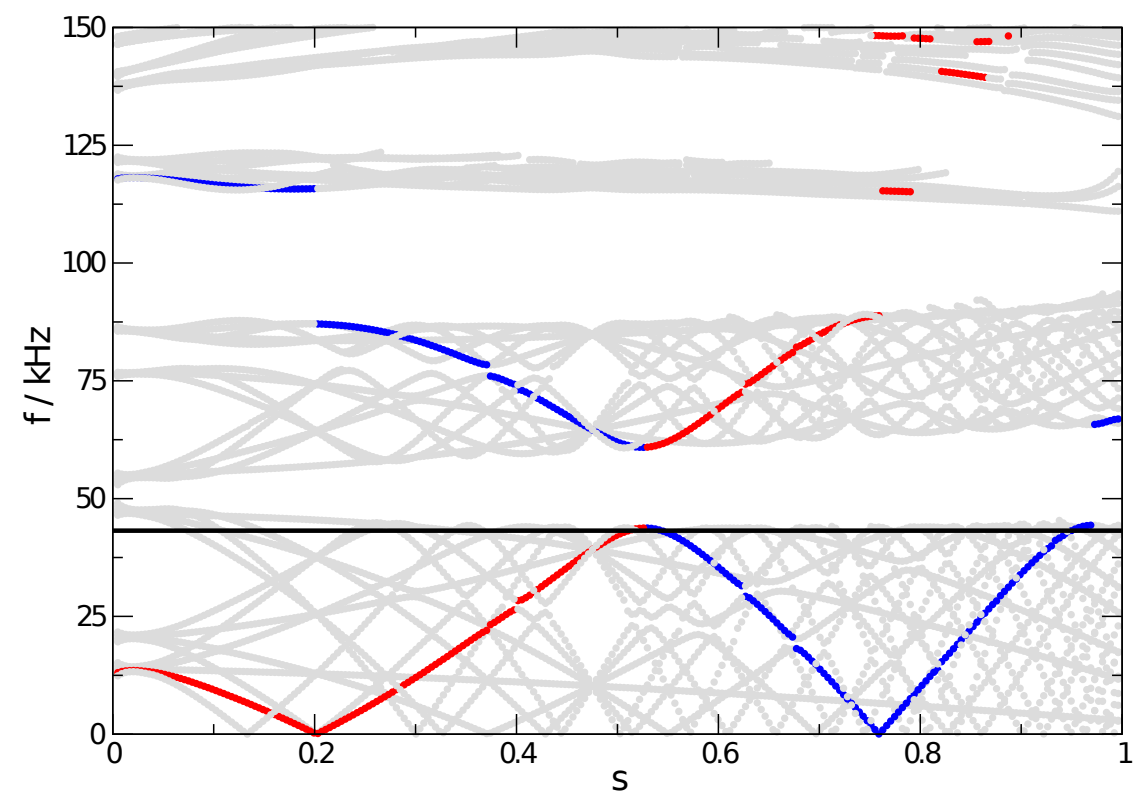

Figure 2: The shear Alfvén continuum for the W7-X high-mirror configuration. While most of the continuum branches are shown in grey, the relevant branches have been coloured. The TAE frequency is shown as a solid black horizontal line and lies at the lower edge of the TAE gap. The mode is radially located near the avoided crossing of the coloured branches.

values of the fast-ion density and the resulting linear growth rates $\gamma_{\mathrm{L}}$ for the different cases (denoted as tokamak case 1, 2, and 3) are given in Tab. I. They all share a common value for the damping rate $\gamma_{d}=1.05 \cdot 10^{4} \mathrm{~s}^{-1}$. This value for $\gamma_{d}$ is chosen, because it is the standard value for the ITPA benchmark case.

For W7-X, a high-mirror configuration is studied. The shear Alfvén wave continuum for this equilibrium is shown in Fig. 2. The relevant continuum branches $(m=17$ in blue and $m=18$ in red) are coloured and the TAE frequency is indicated as a solid horizontal line. The toroidal mode number is $n=-16$. Note that, while the magnetic equilibrium is the same as in Ref. [36], not the same mode is investigated. We follow Ref. [21] and study a slightly more core-localized TAE with higher mode numbers (which leads to a more narrow radial mode structure) that is more readily comparable with the tokamak case. The radial mode structure and fast-ion density profile for W7-X are shown in Fig. 3. For simplicity, the background-plasma density and temperature profiles are flat. We choose $n_{\mathrm{i}}=n_{\mathrm{e}}=10^{20} \mathrm{~m}^{-3}$ and $T_{\mathrm{i}}=T_{\mathrm{e}}=3 \mathrm{keV}$, respectively.

Also for W7-X, two cases are compared with each other. They are denoted as W7-X 

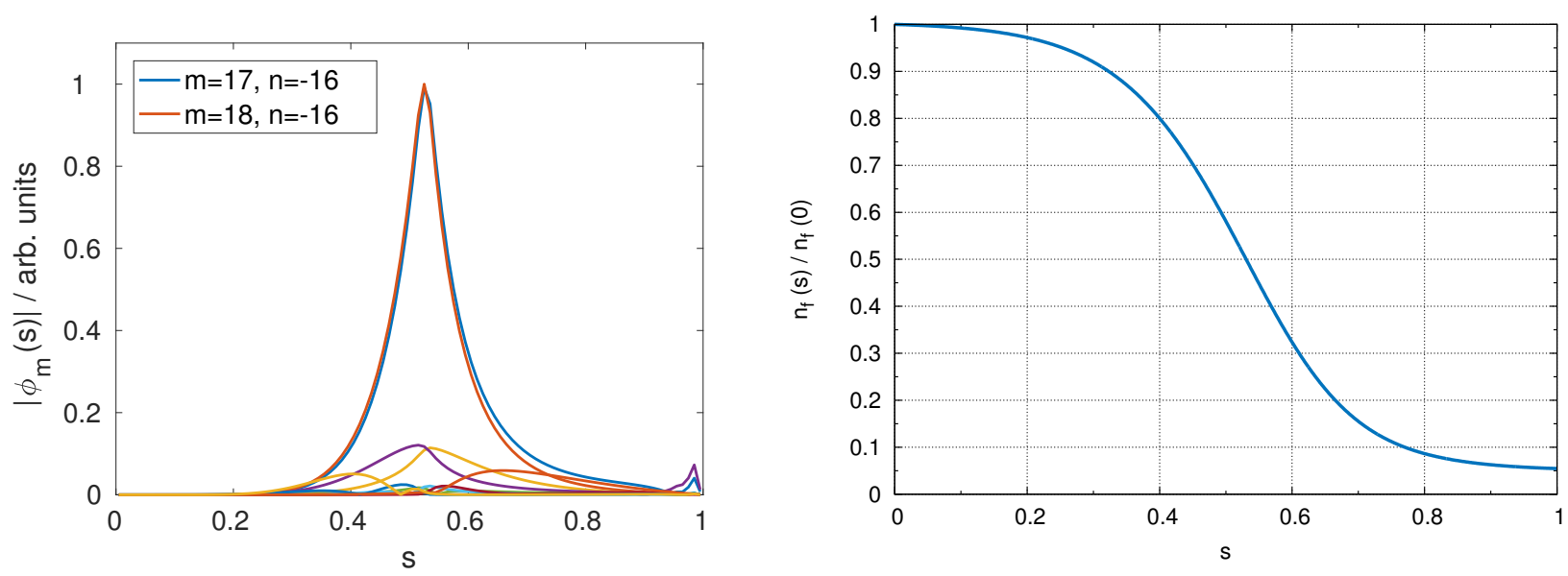

Figure 3: Mode structure (left) and normalized fast-ion density profile (right) for the W7-X case.

Table II: Fast-ion densities and resulting linear growth rates for the W7-X cases.

\begin{tabular}{c|c|c}
\hline \hline case & Density $n_{\mathrm{f}}(0) / \mathrm{m}^{-3}$ & Linear growth rate $\gamma_{\mathrm{L}} / \mathrm{s}^{-1}$ \\
\hline $\mathrm{W} 7-\mathrm{X}$ case 1 & $1.2 \cdot 10^{19}$ & $1.19 \cdot 10^{4}$ \\
\hline $\mathrm{W} 7-\mathrm{X}$ case 2 & $6.0 \cdot 10^{18}$ & $5.90 \cdot 10^{3}$ \\
\hline \hline
\end{tabular}

case 1 and 2. Again, the on-axis values of the fast-ion density as well as the linear growth rates are given in Tab. II. The damping rates are chosen as $\gamma_{\mathrm{d}}=9.6 \cdot 10^{3} \mathrm{~s}^{-1}$ and $\gamma_{\mathrm{d}}=4.8 \cdot 10^{3} \mathrm{~s}^{-1}$ for cases 1 and 2, respectively. Choosing these values is motivated by trying to make the ratio $\gamma_{L} / \gamma_{d}$ similar to the tokamak case 1 . Note that the fast-ion density is very high for the W7-X cases. This is done to make the drive comparable to the ITPA tokamak case in which a high fast-ion temperature of $400 \mathrm{keV}$ is used. Since the fast-ion temperature in W7-X is much lower (see below), the density needs to be increased accordingly.

Note that the velocity-dependent part of the fast-ion distribution function is different for the ITPA case and the W7-X case: We use a Maxwellian distribution function for the ITPA case. The fast particles, which are deuterium ions, have the standard temperature of $T_{\mathrm{f}}=400 \mathrm{keV}$. The fast-ion temperature profile is flat so that the density gradient is the only source of free energy for the instability.

For the W7-X case, on the other hand, a more realistic slowing-down distribution function with three distinct energy levels

$$
f^{(0)}=C \sum_{i=1}^{3} \frac{w_{i}}{v^{3}+v_{\mathrm{c}}^{3}} H\left(v_{\mathrm{b}, i}-v\right)
$$

is used ( $H$ denotes the Heaviside step function). As is well known, not only atomic hydrogen, but also molecules can be accelerated in the NBI system. This leads to the step-like shape of the distribution function, whose parameters (beam velocity levels and corresponding weights) are listed in Tab. III. The highest beam velocity corresponds to an energy of $55 \mathrm{keV}$ of the injected protons. The critical velocity $v_{\mathrm{c}}$ is computed 
Table III: Parameters of the fast-ion distribution function for the W7-X cases.

\begin{tabular}{c|c|c}
\hline \hline$i$ & Velocities $v_{\mathrm{b}, i} / \mathrm{ms}^{-1}$ & Weights $w_{i}$ \\
\hline 1 & $3.28 \cdot 10^{6}$ & 0.398 \\
\hline 2 & $2.34 \cdot 10^{6}$ & 0.347 \\
\hline 3 & $1.92 \cdot 10^{6}$ & 0.255 \\
\hline \hline
\end{tabular}
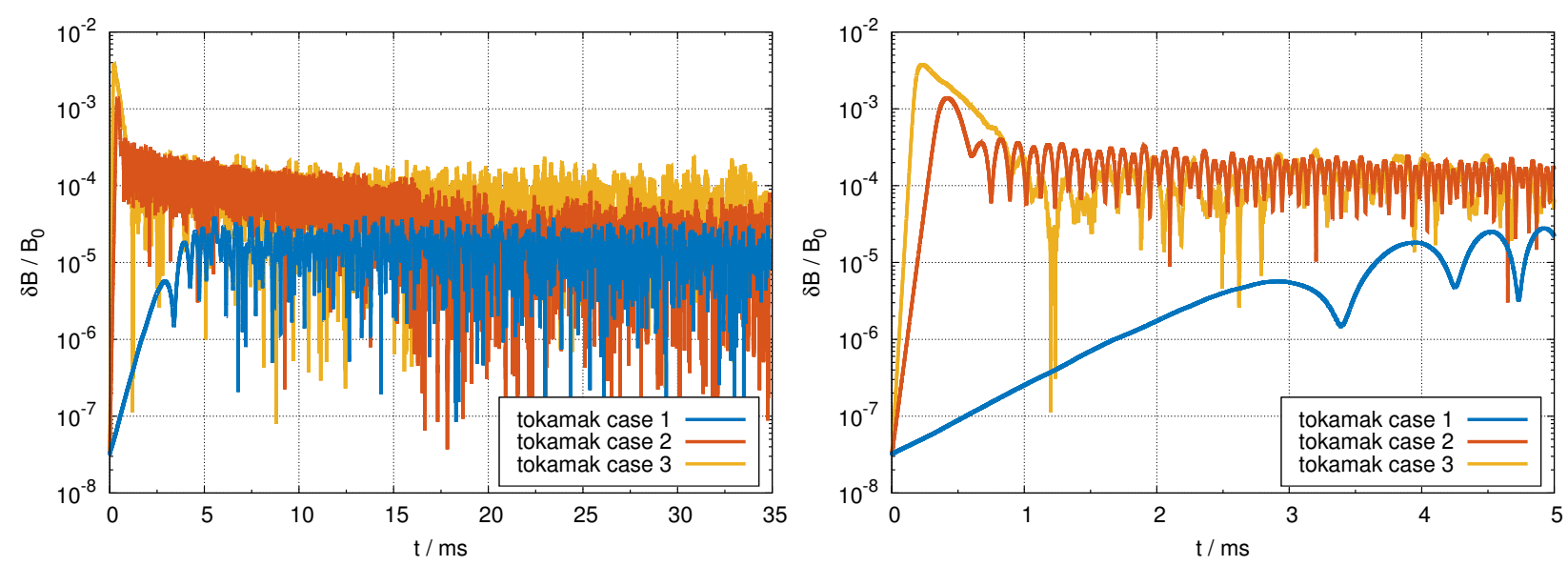

Figure 4: Time traces of the perturbed poloidal magnetic field for the tokamak cases investigated. Calculations are carried out for a long time into the non-linear phase. The left-hand side shows the whole simulation, while the right-hand side shows a zoomed-in view at the time when the initial saturation occurred.

according to Eq. (20) taking into account the temperature and density dependencies. The normalization constant $C$ is determined numerically. Again, the fast-ion density gradient is the source of free energy that drives the instability.

It has to be mentioned here that the collision frequencies are very different for the ITPA case and for W7-X, respectively. To illustrate this fact, we give here the fast-ion selfcollision frequencies (see Eq. (14)) at the respective mode maxima. While for the ITPA case the collision frequency is $v_{0}^{\mathrm{ff}}=2.8 \cdot 10^{-4} \mathrm{~s}^{-1}$, the collision frequency is about four orders of magnitude higher for $\mathrm{W} 7-\mathrm{X}, v_{0}^{\mathrm{ff}}=7.1 \mathrm{~s}^{-1}$. This is due to the higher density and lower temperature of the fast ions.

\section{B. Collisionless results}

All these cases have been chosen because they exhibit frequency chirping in the nonlinear phase of a collisionless simulation. Before showing how collisions can potentially alter the behaviour of the mode in the non-linear phase and how this affects the chirping, we will present the collisionless results for later comparison.

The temporal evolution of the perturbed magnetic field attributed to the mode ( $\delta B$ is defined as global maximum of the perturbed poloidal magnetic field) is shown in Fig. 4 for the ITPA case. Fig. 5 depicts the W7-X high-mirror case. In either case, the simu- 

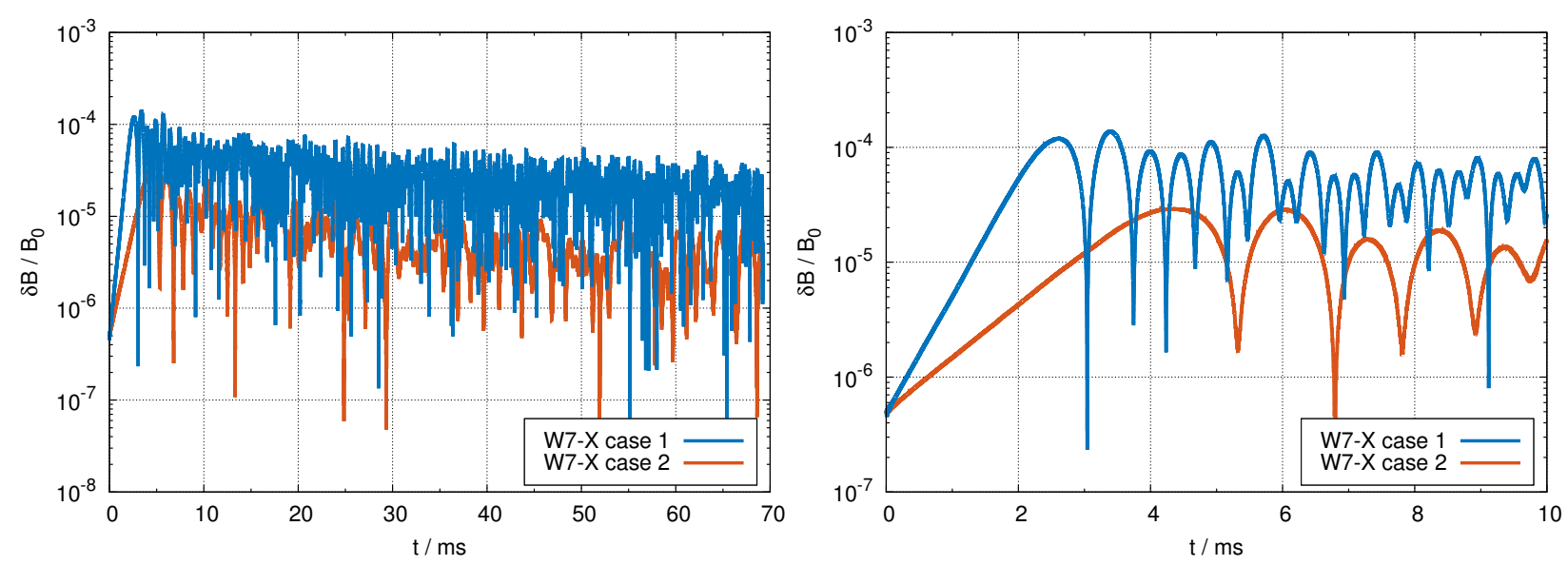

Figure 5: Same as Fig. 4, but for W7-X.
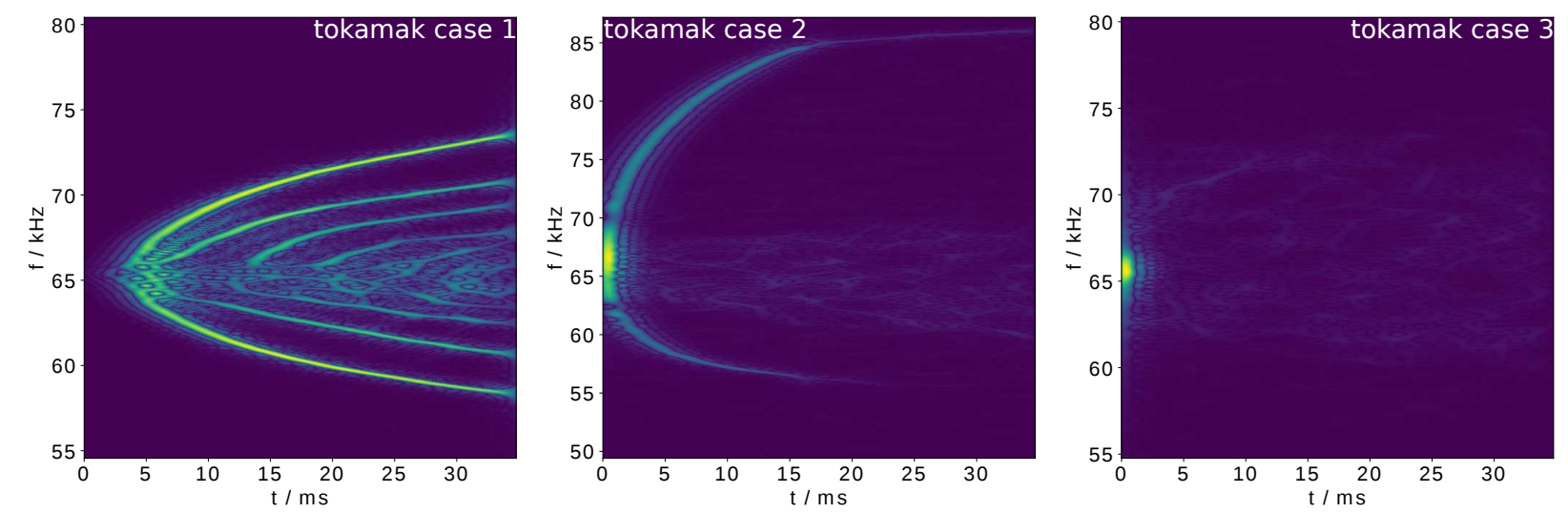

Figure 6: Spectrograms of the perturbed electrostatic potential for the different tokamak cases. The linear growth rate increases from case 1 to 3 . This clearly influences the frequency chirping. While the chirping is only slightly asymmetric for case 1, it becomes very asymmetric for case 2 and has finally completely vanished for case 3.

lations extend for a long time into the non-linear phase. This is necessary in order to capture the frequency chirping with sufficient accuracy. Note that especially for W7-X it is crucial to check the time step for convergence.

The time trace of the perturbed electrostatic potential (whose envelope is directly proportional to $\delta B(t)$ for CKA-EUTERPE) is used to generate the spectrograms shown in Figs. 6 and 7 for the ITPA case and the W7-X case, respectively. Throughout this work the so-called S-method [7] is used to generate the spectrograms. This methods includes a parameter $\lambda$, which can be used to 'interpolate' between a short-time Fourier transform $(\lambda \rightarrow 0)$ and the Wigner distribution $(\lambda \rightarrow \infty)$. For the ITPA case, we use every 5 th value in the time trace of the electrostatic potential and $\lambda=10$ to generate the spectrograms. For W7-X, since the time step is smaller, we use every 10 th value and $\lambda=5$ instead.

Even though the time traces of the perturbed magnetic field (see Figs. 4 and 5) do not look very different in the non-linear phase, the frequency chirping is strikingly different (see Figs. 6 and 7). Symmetric, asymmetric, and vanishing chirping are possible for 

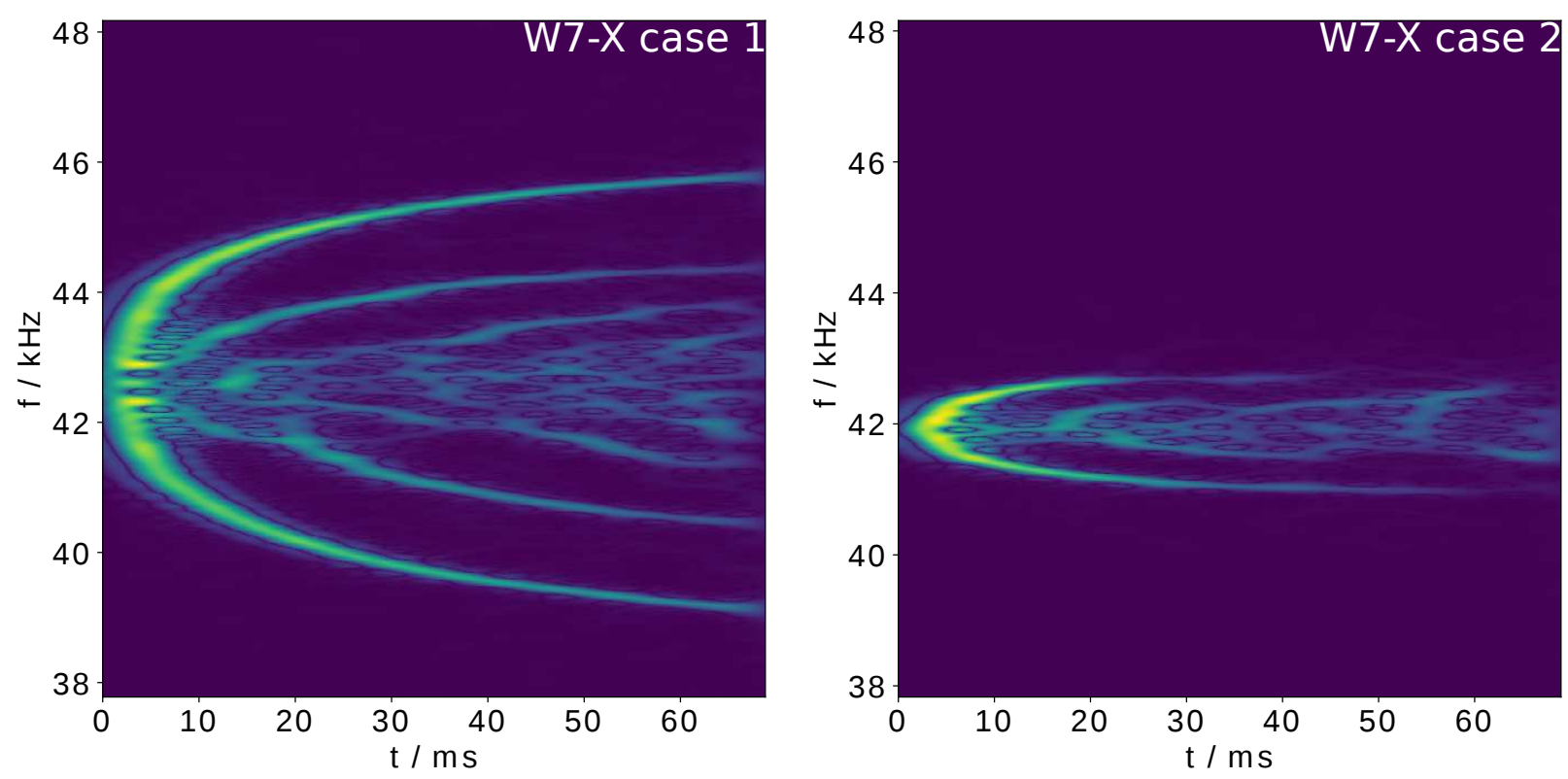

Figure 7: Spectrograms of the perturbed electrostatic potential for the different W7-X cases. Case 1 has twice the linear growth rate and twice the damping rate compared to case 2 . It therefore shows a wider chirping parabola.

both the tokamak and the stellarator. Which chirping scenario is realized depends on the linear growth rate and the damping rate. (That all the cases have a different linear growth rate is clearly visible on the right-hand side of Figs. 4 and 5.) Ref. [21] will discuss the influence of $\gamma_{\mathrm{L}}$ and $\gamma_{\mathrm{d}}$ on the frequency chirping in greater detail.

In the following, we pick only the cases that showed the most symmetric chirping (tokamak case 1 and W7-X case 1 ) in order to assess the influence of different collision operators on the non-linear dynamics.

\section{NON-LINEAR BEHAVIOUR INCLUDING COLLISIONS - ITPA TOKAMAK CASE}

\section{A. Changes induced by pitch-angle collisions}

After having established a solid baseline of what scenarios to expect non-linearly without collisions, we now include pitch-angle collisions in the simulations and investigate how the frequency chirping changes. Different cases are considered: Firstly, we will use the self-consistently calculated self-collision frequency of the fast ions given by Eqs. (13) and (14). Subsequently, in order to emulate the fact that the fast ions do not only collide with themselves, but mainly with background ions and electrons, we will multiply the self-consistent value by 10 and by 100 .

As a representative of the ITPA case, we investigate the symmetric tokamak case 1 . Fig. 8 shows how the time trace of the perturbed magnetic field changes when pitchangle collisions with varying collision frequency are taken into account. The right-hand side of Fig. 8 shows a zoomed-in view on the initial saturation. Several observations 

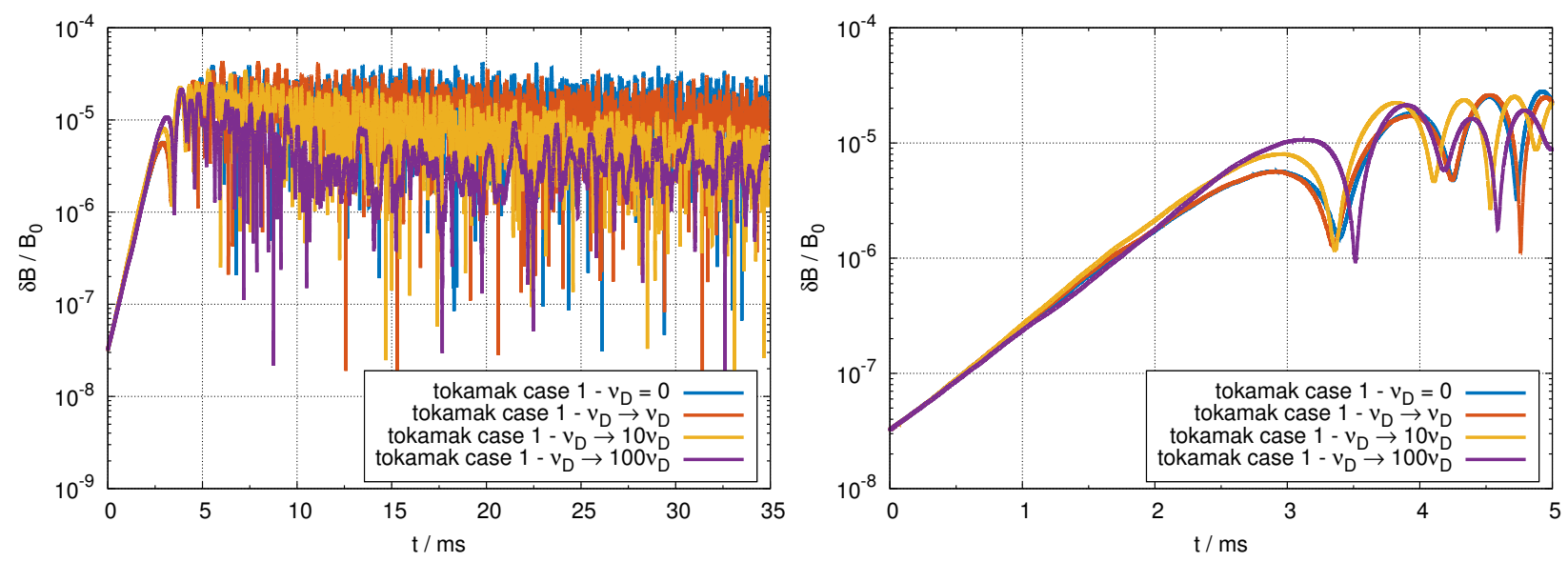

Figure 8: Similar as Fig. 4, but only for tokamak case 1 with a varying pitch-angle collision frequency. $v_{\mathrm{D}}$ denotes the self-consistent value. Higher collision frequencies tend to lead to lower average $\delta B$ values in the long-term non-linear phase. The right-hand side shows a zoomed-in view on the initial saturation.

can be made: Firstly, the linear phases in each simulation are very similar. Therefore, any changes in the frequency chirping can directly be attributed to the presence of the pitch-angle scattering operator and not to changes of $\gamma_{\mathrm{L}}$. Furthermore, as predicted by analytical theory [1] (and numerically confirmed for the same magnetic equilibrium in Ref. [36]), the first maximum of $\delta B$ after the linear phase increases with $v_{D}$ due to the prevention of wave-particle trapping.

The non-linear phases are completely different. Even when the fast-ion self-collision frequency is multiplied by 100 , the collision frequency is still small enough to allow for a periodic non-linear behaviour of the mode. It is striking that even though the initial saturation level (first maximum of $\delta B$ ) increases with $v_{\mathrm{D}}$, this is not true for the long-term non-linear saturation level: It clearly decreases with $v_{\mathrm{D}}$.

Fig. 9 shows the spectrograms for the self-consistent case and two cases with artificially increased collision frequency by either a factor 10 or 100 . Recall that in the standard ITPA tokamak case the fast-ion temperature is high $\left(T_{\mathrm{f}}=400 \mathrm{keV}\right)$. Hence, the fast-ion self-collision frequency is very low. This is reflected in the spectrograms, where the self-consistent case still very closely resembles its collisionless counterpart in Fig. 6. For increased collision frequencies, pitch-angle collisions tend to blur the 'internal structure' within the chirping parabola. Furthermore, they lead to a more narrow chirping in general. Fig. 8 shows that high pitch-angle collision frequencies significantly reduce the mode amplitude for long times, which is also visible in Fig. 9 where the chirping fades out in the high-collisionality case.

\section{B. Changes induced by a fast-ion collision operator}

We will now discuss the influence of a fast-ion collision operator on the non-linear dynamics. Still, a Maxwellian background is used, since the ITPA benchmark is defined that way. 

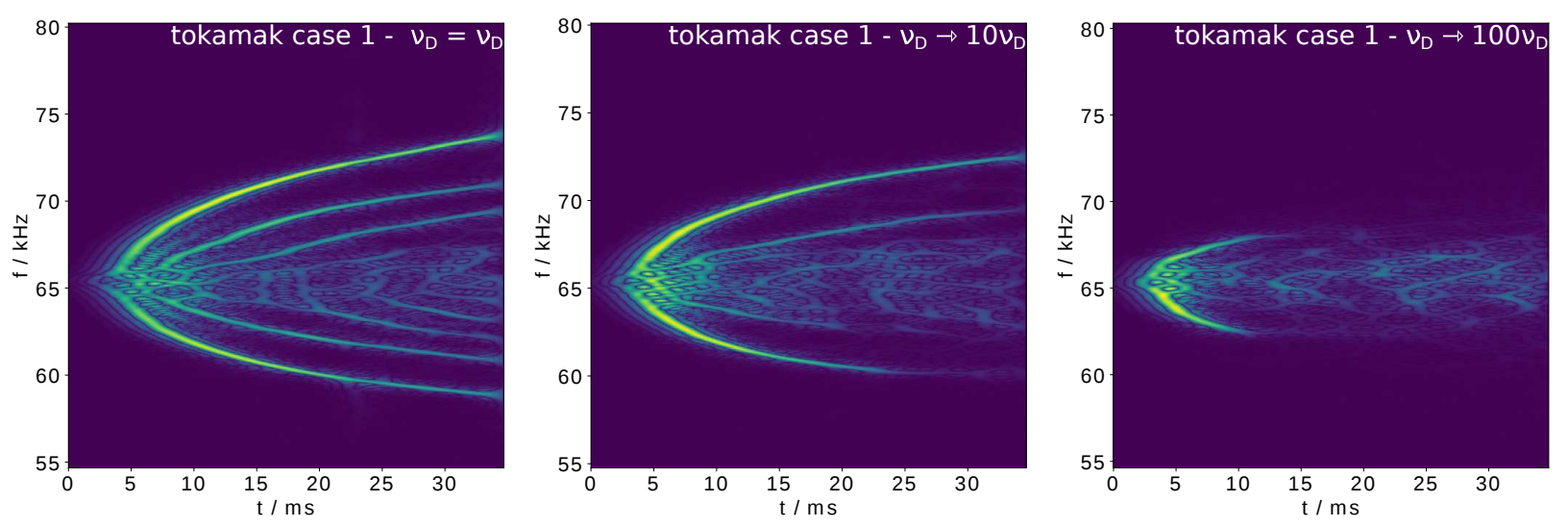

Figure 9: Different non-linear chirping scenarios for tokamak case 1 with varying collisionality (only pitch-angle collisions are considered). The self-consistent value for $\nu_{\mathrm{D}}$ is rather low (fastion temperature is high and density is low), so that the first plot shows no significant deviation from its counterpart in Fig. 6. Higher collision frequencies damp the mode and lead to a more narrow chirping.
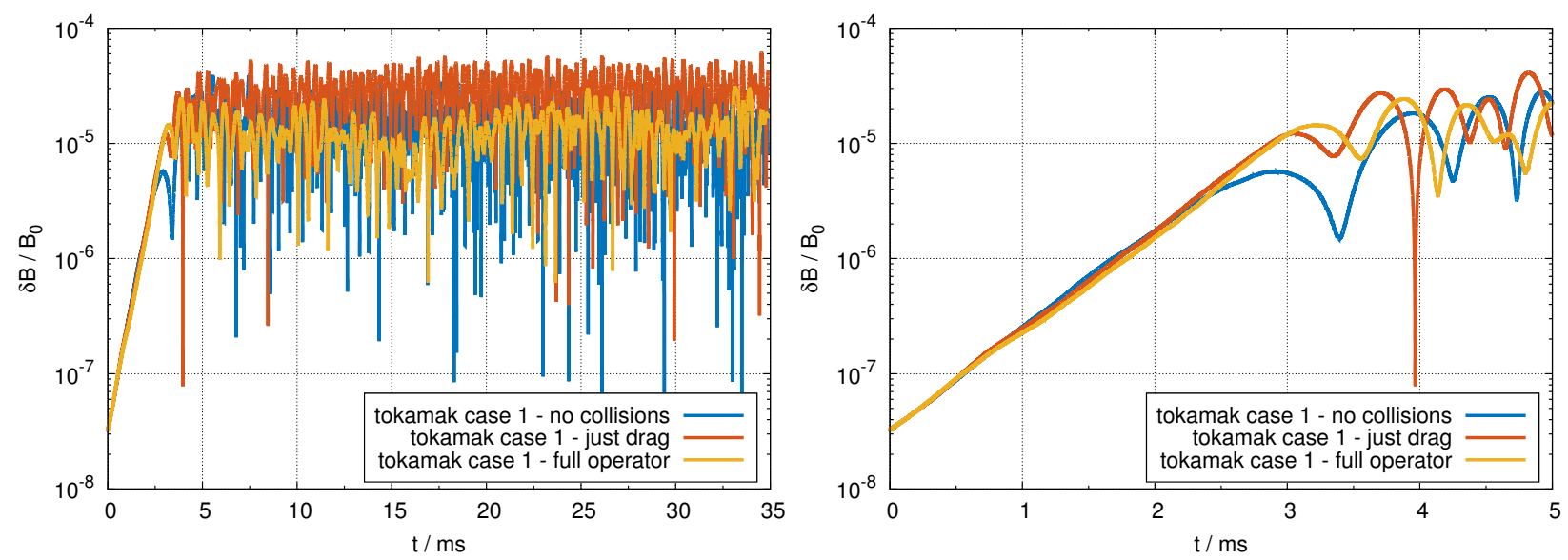

Figure 10: Time traces of the perturbed magnetic field for the reference case without collisions, a case where only the drag-part of the fast-ion collision operator was included, and for the full fast-ion collision operator. The non-linear dynamics is different in each case. The right-hand side shows a zoomed-in view on the time of initial saturation.

Fig. 10 shows how the time trace of the perturbed magnetic field changes when the fastion collision operator (either just drag or the full operator including also pitch-angle collisions) is taken into account. One can see that the non-linear phases are different, both with respect to the long-term averaged saturation level and the period of the nonlinear oscillations. As shown in the expanded view on the right-hand side, the linear phases are still similar. However, the first maximum of $\delta B$ after the linear phase increases due to fast-ion drag. We speculate that the friction force experienced by the fast ions pushes them in and out of the resonance. This could be a mechanism that prevents (or at least impedes) wave-particle trapping. While the addition of pitch-angle collisions in the case of the full fast-ion operator very slightly increases the initial saturation am- 

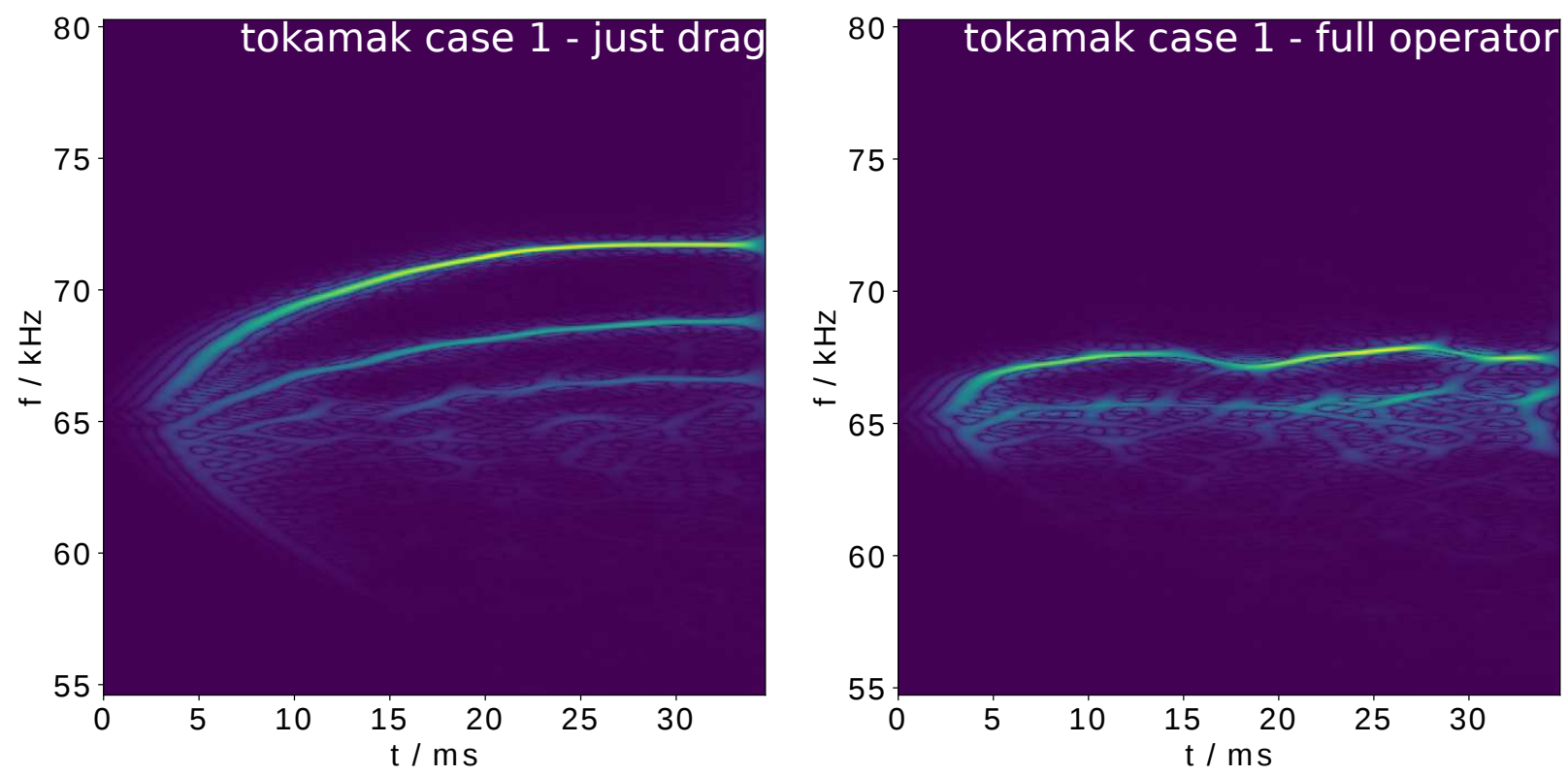

Figure 11: Spectrograms of the perturbed electrostatic potential for a case where just drag is included (left-hand side) and for the full fast-ion collision operator (right-hand side). Comparing with the collisionless result shown on the left-hand side of Fig. 6, we find that, as soon as drag is included, only the up-chirping branches remain.

plitude (first maximum), the just-drag case (without any pitch-angle collisions) shows the highest saturation level for long times.

The spectrograms of the perturbed electrostatic potential, including only drag on the left-hand side and the full fast-ion collision operator on the right-hand side, are shown in Fig. 11. In these spectrograms, the effects of the drag term are visible with their characteristic signature (see, for example, Refs. $[25,29]$ ) and only the branches that show an up-chirping frequency remain. This is due to the fact that the fast-ion collision operator is not symmetric around the resonant velocity. The non-linear frequency evolution changes significantly in the case with the full fast-ion collision operator. As in the dragonly case, the frequency is just (slightly) chirping up, but pitch-angle collisions now lead to a 'modulated' behaviour after some time. As discussed before, they again reduce the width of the chirping parabola. Pitch-angle scattering leads to the de-trapping of particles from the phase-space structure, which reduces the overall chirping. Note that the pitch-angle collision frequency used in this case is close to the $v_{\mathrm{D}} \rightarrow 100 v_{\mathrm{D}}$-case shown in Fig. 9 on the far right. Therefore, the width of the chirping parabola is comparable. Nevertheless, the non-linear behaviour is different in the sense that now the mode amplitude does not decrease for long times, which can only be attributed to the presence of the drag term. 


\section{Using a time-dependent damping rate $\gamma_{\mathrm{d}}(t)$}

It has been studied in the literature (see, for example, Refs. [3, 25, 29]) how the choice of $\gamma_{d}$ influences the non-linear dynamics and frequency chirping. However, these investigations were done with a fixed, but different, value for $\gamma_{d}$ in each simulation. Here, we investigate the influence of a time-dependent damping rate $\gamma_{d}(t)$ on the non-linear dynamics in a single simulation.

The reason for this investigation is that two effects have to be distinguished: Firstly, the frequency of the mode may change on a short time scale, much shorter than the evolution of the equilibrium. This is the effect that is traditionally referred to as chirping. Secondly, the equilibrium may change on a longer time scale. Such equilibrium changes could, for instance, lead to a change in the shear Alfvén continuum gap structure, which could influence $\gamma_{L} / \gamma_{d}$, a critical parameter that determines the frequency chirping. Distinguishing these effects is important, especially for future comparisons with experimental measurements. Below, we use a time-dependent damping rate in order to simulate possible equilibrium changes.

We perform the substitution $\gamma_{\mathrm{d}} \rightarrow \gamma_{\mathrm{d}} f(t)$ in the amplitude equations of CKA-EUTERPE (see Eqs. (7) and (8)) with a time-dependent function

$$
f(t)=-\varepsilon_{\gamma_{\mathrm{d}}} \sin \left[\omega_{\gamma_{\mathrm{d}}}\left(t-\Delta t_{\gamma_{\mathrm{d}}}\right)\right]\left(\frac{\operatorname{erfc}\left[t-\Delta t_{\gamma_{\mathrm{d}}}\right]}{2}-1\right)+1 .
$$

The parameters in $f(t)$ are chosen as

$$
\begin{aligned}
\varepsilon_{\gamma_{\mathrm{d}}} & =0.8 \\
\omega_{\gamma_{\mathrm{d}}} & =430.1 \mathrm{~s}^{-1} \\
\Delta t_{\gamma_{\mathrm{d}}} & =3.14 \mathrm{~ms} .
\end{aligned}
$$

With this choice of parameters, $\gamma_{d}$ will not vary in the linear phase. This ensures that the linear growth rate will be the same as in the regular collisionless reference case. This way, any changes in the non-linear phase cannot have their origin in a different linear phase. The resulting time trace of the perturbed magnetic field, including the time-dependent damping rate, is shown in Fig. 12. The figure shows that, in the nonlinear phase, the average value of $\delta B$ now oscillates with $\gamma_{\mathrm{d}}(t)$. Even when $\gamma_{\mathrm{d}}(t)$ is at its maximum (see maxima of red curve), the mode does not return to its initial value so that a new linear phase may start.

Analytical theory $[3,25]$ predicts that the frequency change scales as $\delta \omega(t)=\omega(t)-$ $\omega(0)= \pm \alpha \gamma_{\mathrm{L}} \sqrt{\gamma_{\mathrm{d}} t}$ with $\alpha \approx 0.44$ (for the one-dimensional Berk-Breizman paradigm). Substituting the parameters of the collisionless reference case, $\gamma_{L}=\gamma+\gamma_{d}=1.25$. $10^{4} \mathrm{~s}^{-1}, \gamma_{\mathrm{d}}=1.05 \cdot 10^{4} \mathrm{~s}^{-1}$, and $\alpha=0.25$, one arrives at the red curves in Fig. 13 (lefthand side). One can see that the fit works rather well for the upper branch for the case where $\gamma_{d}$ is constant. The fit is worse for the lower branch, since the chirping parabola is slightly asymmetric. When $\gamma_{d}$ changes over time, the analytical theory does not apply. This may be due to the fact that one of the assumptions of the Berk-Breizman model, that the mode is close to marginal stability, is violated when $\gamma_{\mathrm{d}}(t)$ is at its minimal values. Hence, the theory predicts a non-monotonic behaviour of $\delta \omega$, whereas we find a slightly modulated, but always monotonic behaviour in the simulations. 

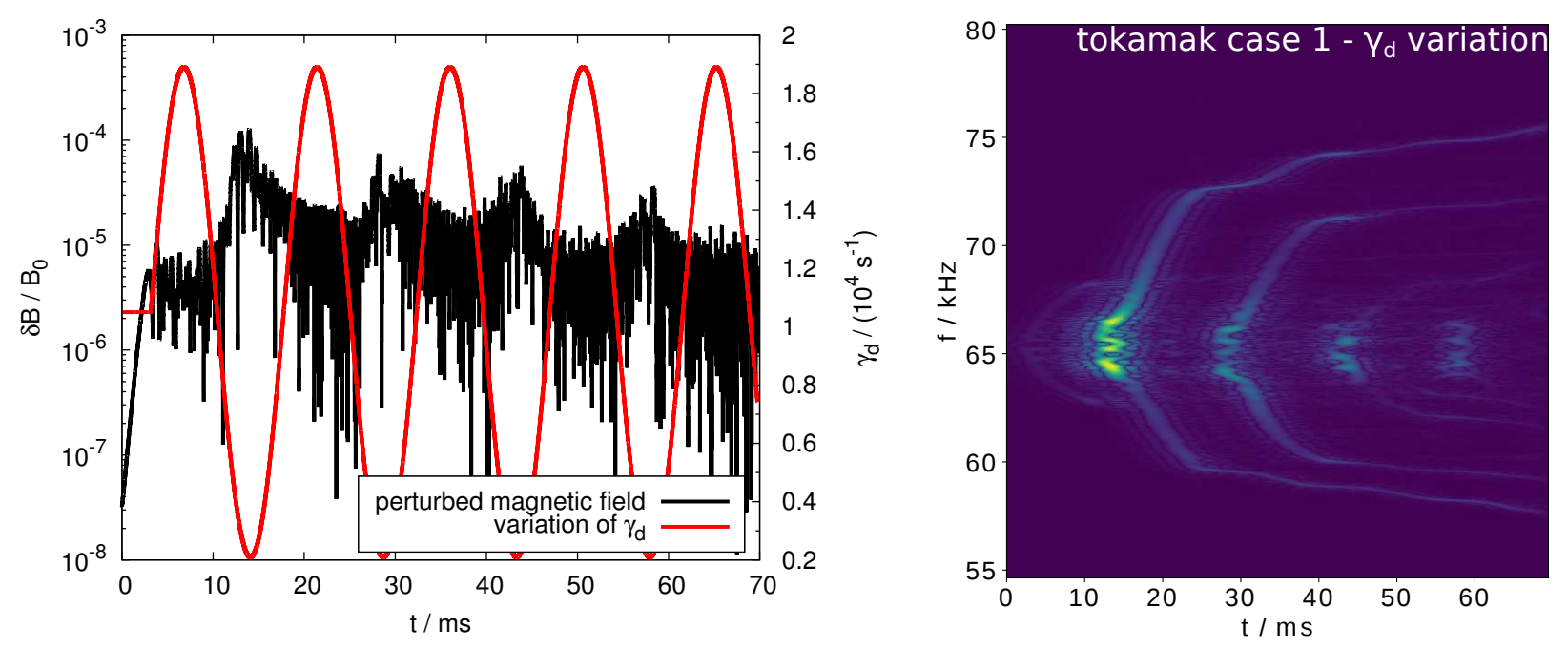

Figure 12: Temporal evolution of $\gamma_{\mathrm{d}}$ and the induced temporal behaviour of the perturbed magnetic field (left-hand side). The right-hand side shows the spectrogram of the perturbed electrostatic potential, which is now also modulated (parabola is either narrow or wide) with the same period as $\gamma_{\mathrm{d}}$.
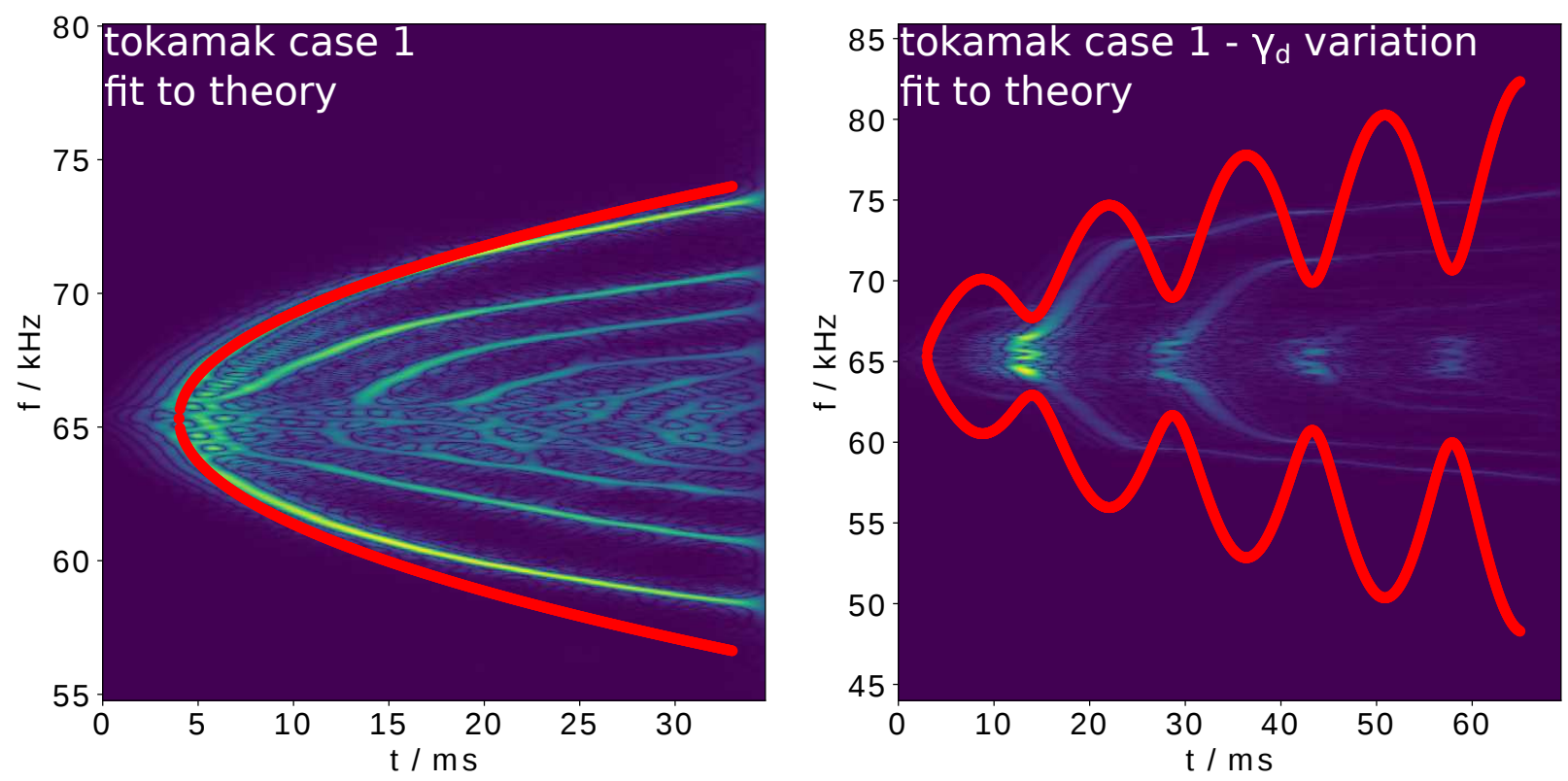

Figure 13: Fits of $\delta \omega(t)=\omega(t)-\omega(0)$ using analytical theory [3, 25]. The standard case is shown on the left-hand side. While the upper branch is fitted rather well, the chirping is slightly asymmetric so that the fit works less well for the lower branch. The right-hand side shows the case in which $\gamma_{d}$ is not a constant. For a time-dependent $\gamma_{d}(t)$, the analytical theory fails to predict the numerically observed behaviour. 

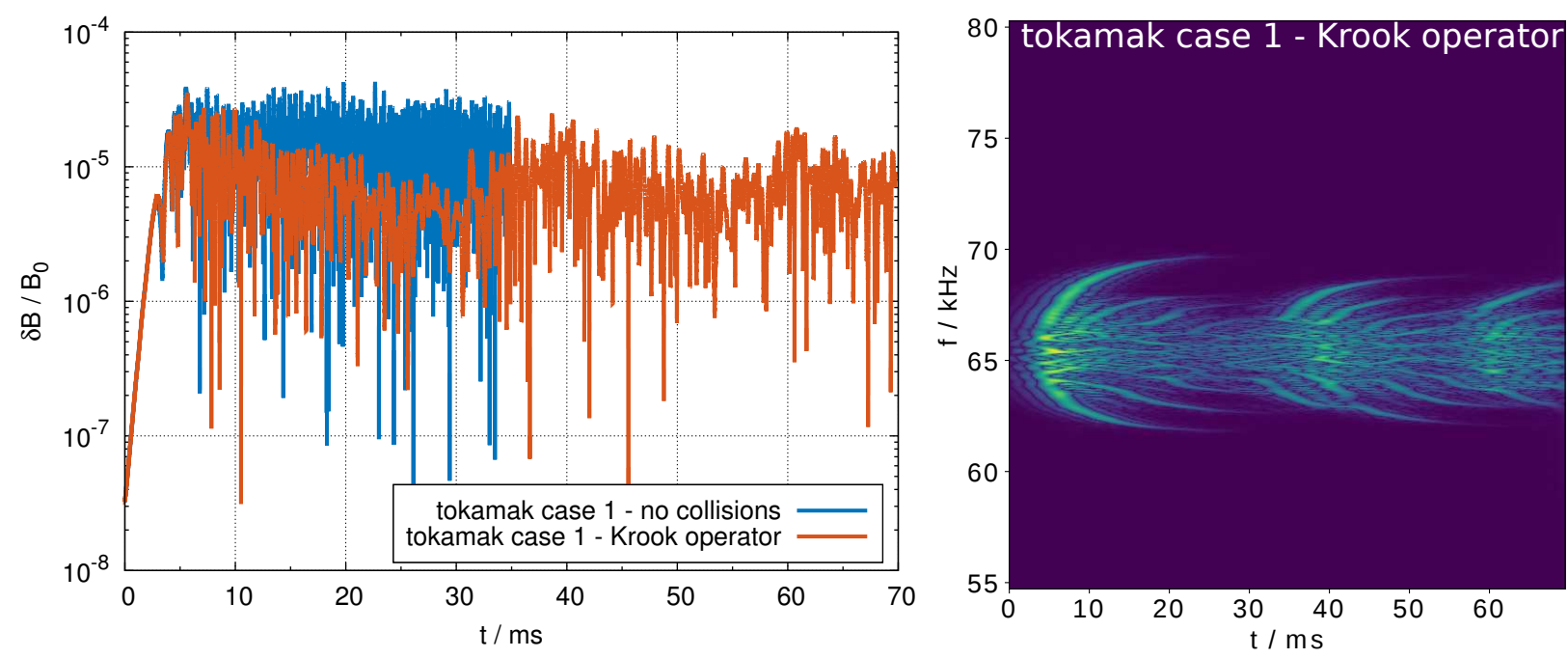

Figure 14: Influence of a Krook operator on the non-linear dynamics. The left-hand side shows the time trace of the perturbed magnetic field. The Krook operator periodically rebuilds the distribution function such that $\delta B$ becomes modulated. The effect on the chirping can be seen on the right-hand side. The Krook operator leads to periodic chirping events.

\section{The influence of a Krook operator}

We now study the influence of a Krook operator on the non-linear dynamics. Since this operator acts to rebuild the initial distribution function, it should lead to periodic chirping events.

It was shown in Ref. [30] that such events are linked to a local flattening of phase-space gradients, followed by the emission of holes and clumps [4], and finally a restoration of the initial gradient so that the process can repeat again. Note that our case is different from the simple Berk-Breizman paradigm: In the Berk-Breizman model a gradient in velocity space is the source of the instability. After this gradient in velocity space is flattened non-linearly, pitch-angle collisions (acting in velocity space) can rebuild it and thus restore the initial distribution function. In the present case, instead of velocityspace gradients, the spatial fast-particle density gradient is the source of instability. We found previously in Sec. IV A that pitch-angle collisions cannot (sufficiently) rebuild the gradient of the density profile and therefore we do not observe periodic chirping. What would be needed is a source of particles in real space. Therefore, a Krook operator is now used to emulate such a source (as would be present in a real experiment via e.g. neutral beam injection), which acts to restore the distribution function to its initial value. This leads to a periodic re-appearance of the mode with intermediate phases of lower mode amplitude, which is a common feature of non-linear systems in general. For instance, the fishbone burst cycle [8] is closely related.

Fig. 14 shows the resulting non-linear dynamics for $v_{\mathrm{Krook}}=114.7 \mathrm{~s}^{-1}$. This value is chosen since it should guarantee several chirping events during a 60-ms-long simulation. Even though the Krook operator is present from the beginning of the simulation, it does not significantly change the first maximum of $\delta B$, frequency and growth rate of the 

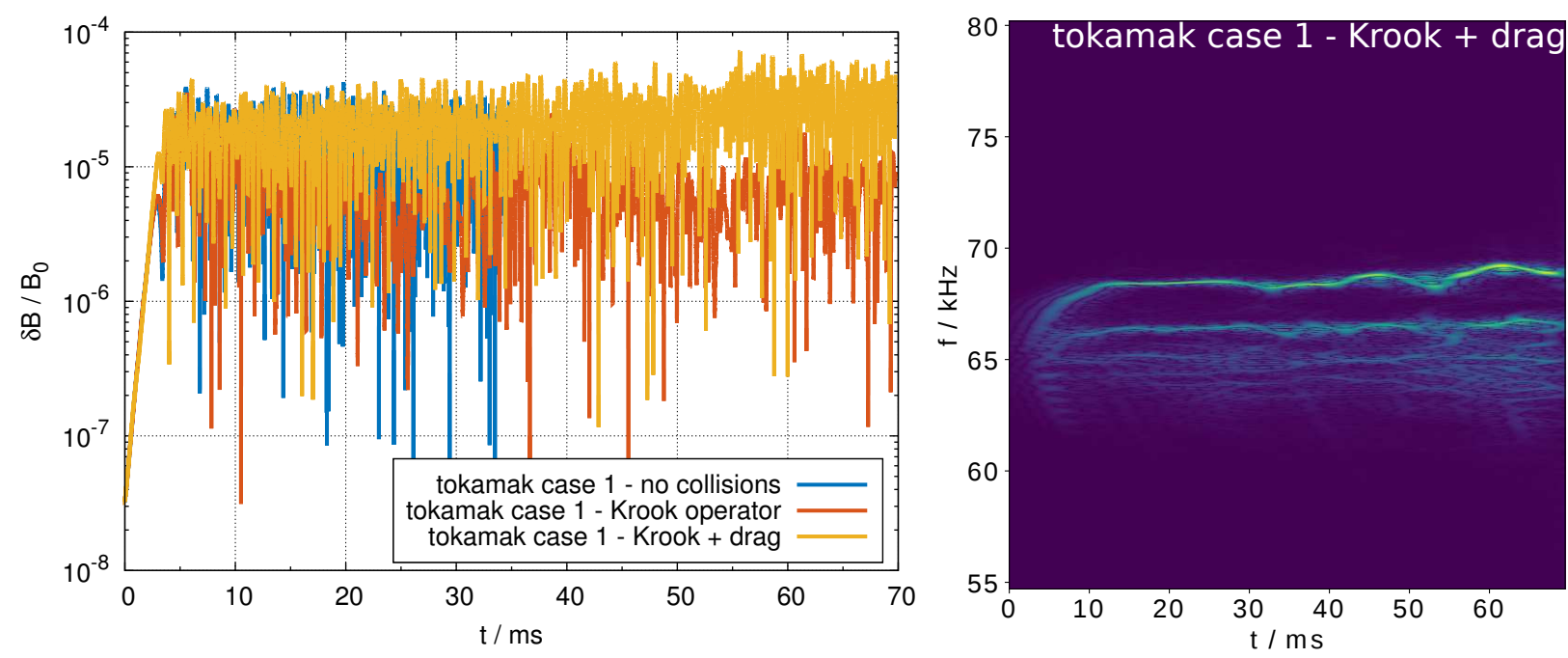

Figure 15: Influence of both Krook collisions and fast-ion drag on the non-linear dynamics. As before, the left-hand side shows the time trace of the perturbed magnetic field. When the drag term is included, the mode amplitude rises on average and is no longer modulated (even though the Krook term is still present). On the right-hand side, which shows the spectrogram of the perturbed electrostatic potential, the drag term now prevents individual, periodic events. The chirping is more narrow.

mode compared with the reference case. (For example, the linear growth rate is reduced by less than $0.1 \%$.) Clearly, the presence of the Krook operator lowers the long-term saturation level. But, as expected, it also leads to a periodic modulation of $\delta B$ and therefore to periodic chirping events as can be seen on the right-hand side of Fig. 14 . The initial chirping parabola fades away such that individual chirping events become visible. The average period in between the major chirping events is roughly $27.5 \mathrm{~ms}$. This is about three times longer than $v_{\text {Krook }}^{-1}$, which indicates that in this complex system other processes, such as the particle motion itself, influence the rate of construction of the distribution function. In the Berk-Breizman model, the Krook operator can be connected to the diffusive processes taking place at the phase-space resonance surface separating the fast ions trapped by the wave from passing particles. In that case, $v_{\mathrm{Krook}}$ should reflect the characteristic de-trapping frequency of fast ions.

It may now be attempted to combine the Krook operator with a fast-ion collision operator. We already showed (see also Fig. 11) that the pitch-angle-scattering part of the full fast-ion collision operator leads to a narrowing chirping parabola. Otherwise, pitchangle collisions do not significantly change the non-linear behaviour. Therefore, we restrict ourselves to the combination of the Krook operator and the drag term. The results can be seen in Fig. 15. As observed before (see Fig. 10), the inclusion of drag leads, on average, to a rising mode amplitude in the non-linear phase. Somewhat surprisingly, the long-term oscillations in the signal - induced by the presence of the Krook term that acts to rebuild the distribution function - are no longer present when fast-ion drag is included. We may again speculate that this is because particles are shifted in and out of the resonance as the simulation progresses. The absence of these long-term non-linear 
oscillations also manifests itself in the spectrogram (see right-hand side of Fig. 15). The chirping does no longer 'tear off' in such a way that individual events are visible. On the other hand, the drag term presents itself with its usual signature (only up-chirping remains). After chirping initially, the frequency stays roughly constant in the non-linear phase. Ref. [33] argues that this corresponds to a state in which the effects of drag and relaxation of the distribution function due to the Krook term, balance each other.

\section{NON-LINEAR BEHAVIOUR INCLUDING COLLISIONS - WENDELSTEIN 7-X CASE}

The stellarator cases have been introduced in Sec. III. We will only focus on W7-X case 1 as it nicely showed almost symmetrical frequency chirping in the absence of collisions. Now we will investigate how the non-linear dynamics changes when particle collisions are included.

\section{A. Changes induced by pitch-angle collisions}

We now investigate for W7-X how pitch-angle collisions between the fast particles influence the frequency chirping and the non-linear dynamics in general. The results are compared to the collisionless reference case. The self-consistently calculated pitch-angle collision frequency $v_{\mathrm{D}}$ is given by Eqs. (13) and (14). Since for W7-X the fast-ion temperature is much lower than for the ITPA tokamak case (and, simultaneously, the fast-ion density is much higher), the collision frequency is also larger. (Recall that we use a slowing-down distribution function. The temperature is therefore computed by calculating the pressure from the distribution function and then dividing by the density. Note that a Maxwellian with roughly the same shape as the slowing-down distribution function would have an equivalent temperature of approximately $20 \mathrm{keV}$.) The effect of the high collision frequency is, as can be seen in Fig. 16, that a steady-state develops after the initial saturation. This is in line with Refs. $[35,36]$ were it was shown recently that a steady-state develops in the non-linear phase for high collision frequencies. Further simulations are performed at one tenth and one hundredth of the original value of the collision frequency. The results are summarized in Fig. 16. The figure shows the time traces of the perturbed magnetic field for the reference case and for the collisional cases. The long-term saturation level is periodic only for the collisionless case. For the lowest collision frequency investigated, the behaviour is still close to being periodic. In that case, however, the damping of the wave in the early non-linear phase is severe. The first maximum of $\delta B$ increases with increasing collision frequency $\nu_{\mathrm{D}}$ (see Fig. 16 right-hand side).

Fig. 17 shows the associated spectrograms of the perturbed electrostatic potential for the cases including collisions. (The collisionless reference case is depicted in Fig. 7 on the left-hand side.) Note that the spectrograms contain less data for the collisional cases, since the time simulated is much shorter. Recall that the collisionless reference case nicely shows almost symmetric chirping (although, comparing $\delta \omega / \omega$, on a much smaller level than the ITPA case). However, as soon as collisions are added, the frequency basically becomes constant in time (see Fig. 17). Only for the lowest collision 

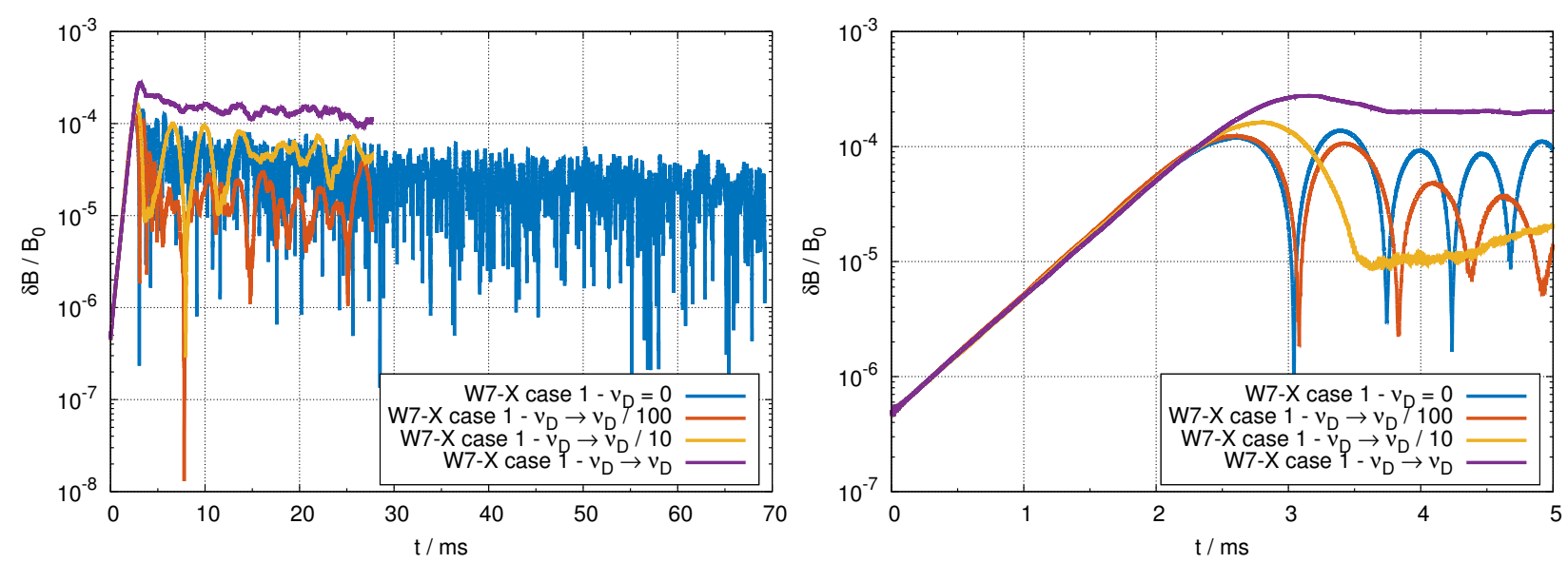

Figure 16: Time traces of the perturbed magnetic field of a TAE mode in Wendelstein 7-X geometry. The pitch-angle collision frequency is varied. $v_{\mathrm{D}}$ denotes the self-consistent value. Except for the lowest collision frequency at early times, the non-linear dynamics resembles a steady-state when collisions are added. Contrary to the ITPA case, higher collision frequencies lead to a higher long-term-average saturation level. The right-hand side shows a zoomed-in view on the initial saturation.
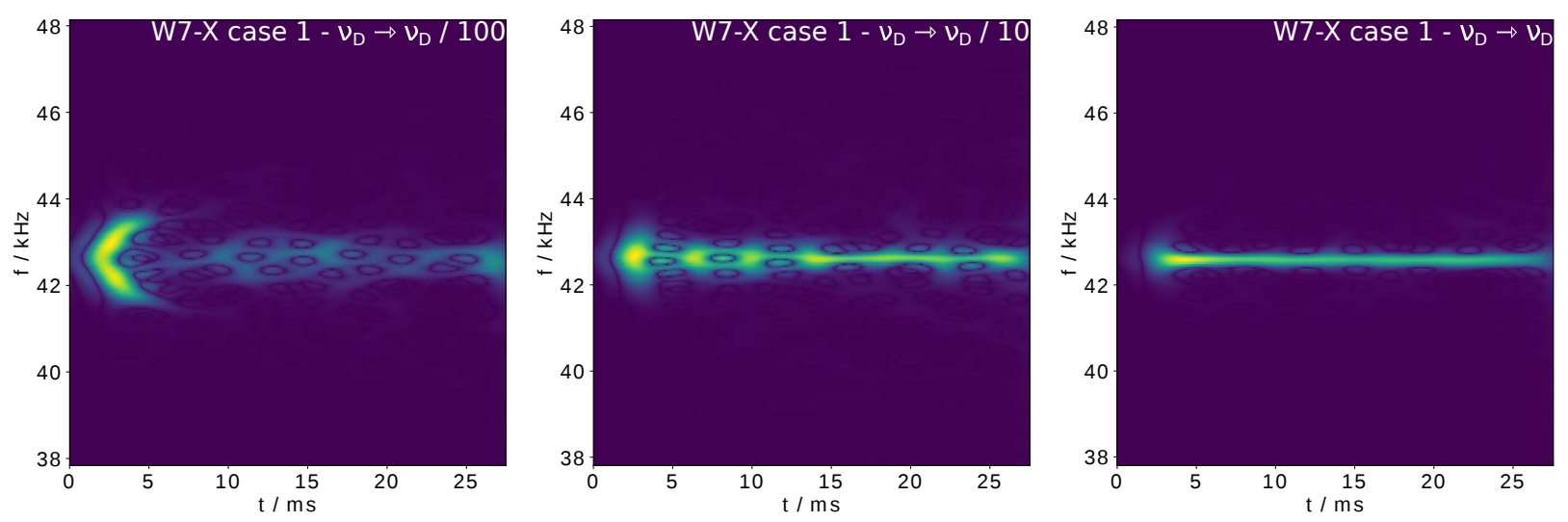

Figure 17: Effect of pitch-angle collisions on the non-linear chirping behaviour for the W7-X case 1 . The self-consistent value for $v_{\mathrm{D}}$ is high (low fast-ion temperature), so that the mode frequency becomes constant. Reducing the collision frequency (left-most sub-figure) leads to a re-appearance of the initial chirping, but significantly damps the mode.

frequency in this set (the left-most sub-figure) are traces of frequency chirping still visible in the early non-linear phase. Nevertheless, because the mode amplitude strongly decreases after the linear phase (see Fig. 16), the chirping eventually disappears.

\section{B. The influence of fast-ion drag}

It is a major goal of the W7-X physics program to assess the influence of fast particles on plasma performance, especially since good fast-ion confinement is an optimization criterion of $W 7-X[12,17]$. A realistic treatment of the fast ions requires not only realistic 

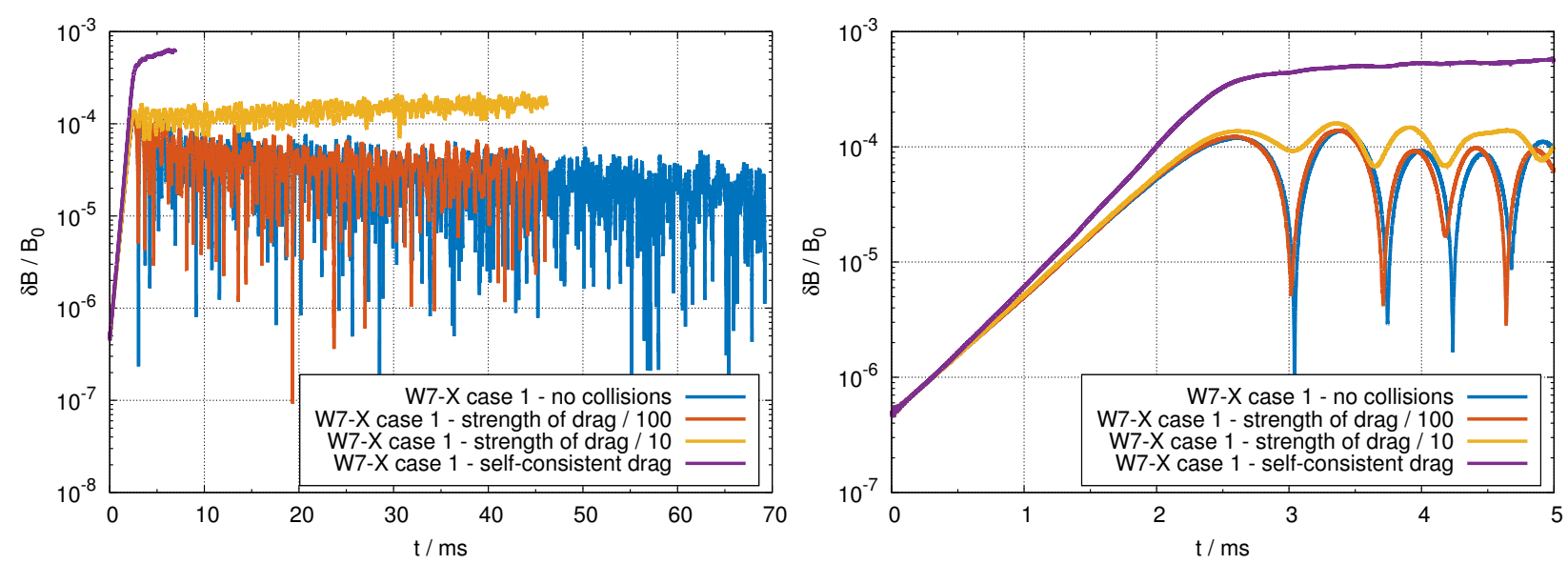

Figure 18: Time traces of the perturbed magnetic field for the W7-X case including a drag term of various strengths. The self-consistent case shows a very high saturation level. Reducing the strength of the drag term brings the curves closer to the collisionless reference. The right-hand side shows a zoomed-in view on the time of initial saturation.

distribution functions, but also the inclusion of a fast-ion collision operator. This is in particular important for non-linear simulations as they are performed here. As described in Sec. II, the fast-ion collision operator combines pitch-angle collisions and drag. Since the influence of pitch-angle collisions has already been addressed, we will now focus on drag only.

The effect of fast-ion drag on the frequency chirping in tokamaks was already discussed in Sec. IV B. This section aims at showing the differences and similarities in stellarators. The slowing-down distribution function described by Eq. (24) is the steady-state solution of the gyro-kinetic equation including a fast-ion collision operator and a beam-like fastion source. The formation of such a distribution function happens on a time scale that is much longer than the Alfvén wave dynamics. Therefore, we use the aforementioned Eq. (24) as a static background and simulate only the deviations from this distribution function caused by the presence of the mode.

The time traces of the perturbed magnetic field, while varying the strength of the drag term, are shown in Fig. 18. If the fast-ion drag is computed self-consistently, the nonlinear saturation level is increased substantially compared with the collisionless reference case. This strong influence of the drag term is, again, due to the high collision frequencies in W7-X compared with the tokamak case. Note that self-consistently calculated drag quickly leads to a steady state with no signs of periodic behaviour. It is therefore excluded from further analysis.

Reducing the drag term by a factor of 10 or 100 leads to results that preserve the chirping nature of the solution in the non-linear phase. After the mode has initially saturated, there is still a sub-exponential drift. The same behaviour was seen before in the tokamak case.

The frequency spectrograms associated to these time traces are shown in Fig. 19. They show the expected behaviour: The chirping becomes asymmetric with the upper branch dominating in intensity over the lower branch. On the right-hand side of the figure, where the strength of the drag term is higher, the lower branch vanishes completely and 

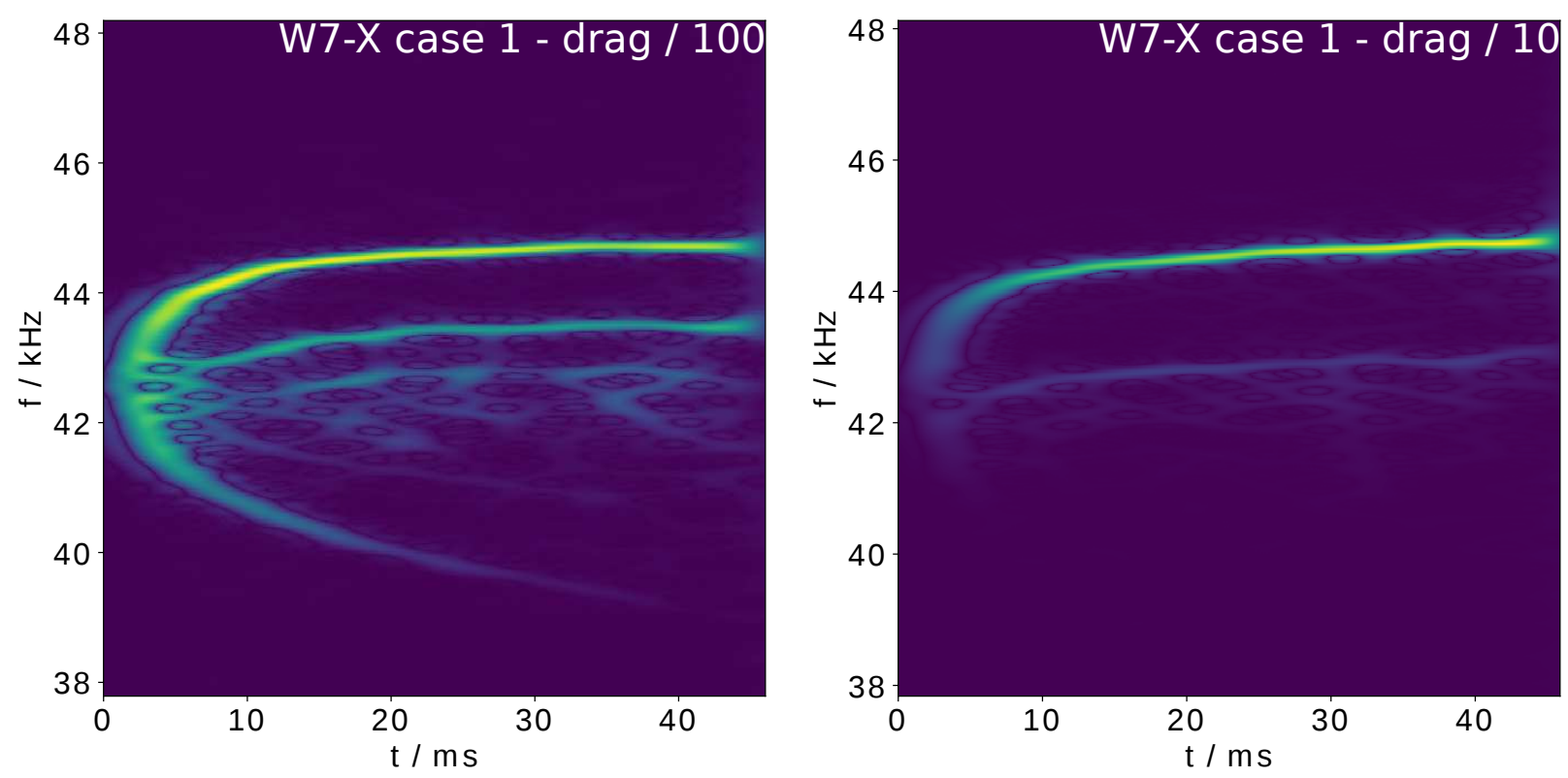

Figure 19: Spectrograms of the perturbed electrostatic potential for the W7-X cases that include fast-ion drag. Compared with Fig. 18, only the cases with reduced strength of the drag term are shown as they preserve the chirping behaviour. As before in the tokamak, drag leads to the disappearance of the down-chirping branches.

the upper branch, after chirping initially, transitions to a new steady state. This is very similar to Fig. 14 of Ref. [29], even though the dimension-less parameters that determine the frequency chirping (normalized growth rate and collision frequencies) are different in this publication.

\section{The influence of a Krook operator}

Finally, we want to demonstrate the effect of a Krook operator in stellarator geometry. We choose $v_{\text {Krook }}=86.7 \mathrm{~s}^{-1}$. The results are shown in Fig. 20. The linear phase remains nearly unaffected (the growth rate changes by less than $0.05 \%$ ). As before in the tokamak case (see Fig. 14 for comparison) the average saturation level in the nonlinear phase is lower when the Krook operator is included. This is most clearly visible up until $t \cong 20 \mathrm{~ms}$. Especially in the late non-linear phase $(t>30 \mathrm{~ms})$ a modulation of the $\delta B$ signal due to the presence of the Krook term is visible. These modulations translate into individual chirping events as shown in the spectrogram on the right-hand side of Fig. 20. The average period between the major chirping events is approximately $19.7 \mathrm{~ms}$, which is about twice $v_{\text {Krook}}^{-1}$. It has to be pointed out that the reduction of the average saturation level and the modulation of the $\delta B$ signal is less pronounced compared with the tokamak case. Note, furthermore, that this one choice of $v_{\text {Krook }}$ corresponds to a proof-of-principle calculation aimed at showing that periodic chirping is possible in W7-X. Details about this process (e.g. how the time in between chirping events in influenced by $\gamma_{\mathrm{L}}$ and $v_{\text {Krook }}$ ) will be investigated in a future publication. 

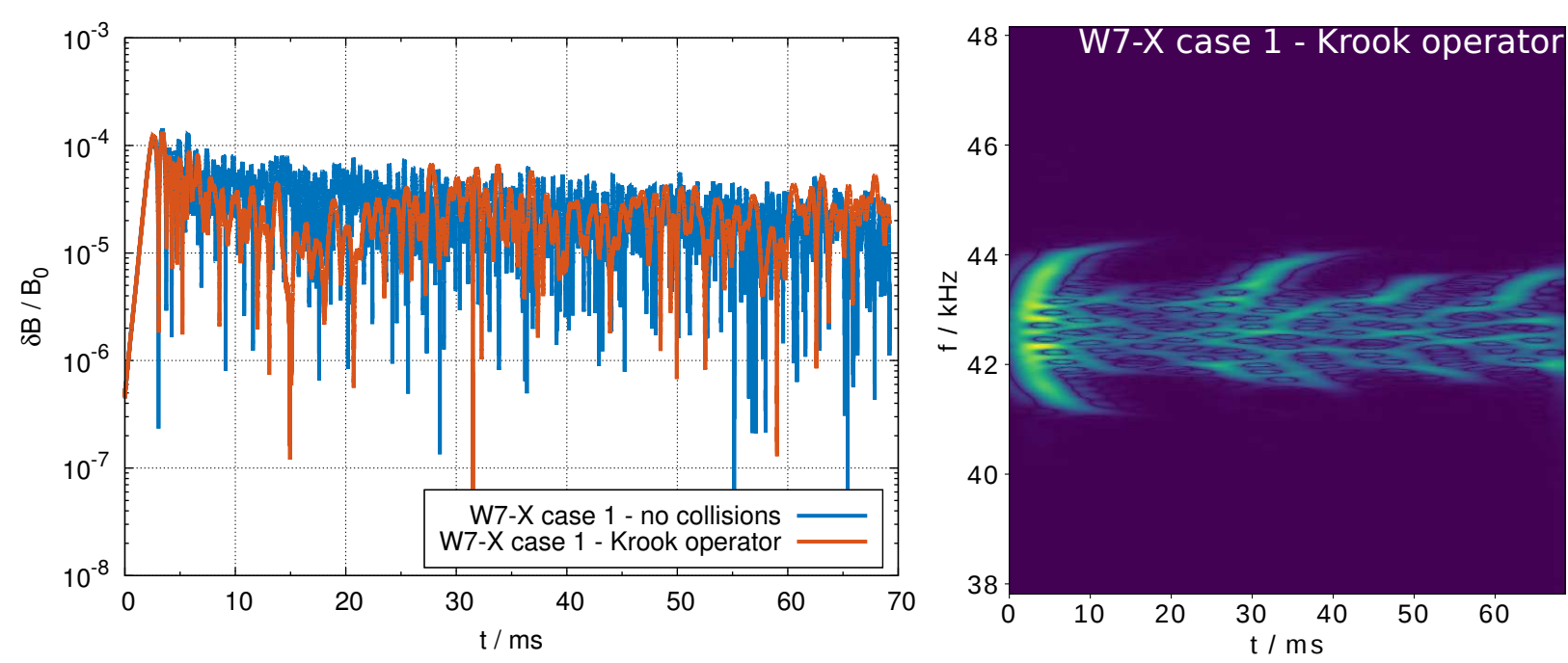

Figure 20: Influence of a Krook operator on the non-linear dynamics of a TAE in W7-X. The left-hand side shows the time trace of the perturbed magnetic field. As for the tokamak case, the Krook operator leads to a periodic modulation of $\delta B$ via rebuilding of the distribution function. In the spectrogram on the right-hand side the Krook operator leads to periodic (and separated) chirping events.

\section{SUMMARY AND CONCLUSIONS}

In this paper, we have investigated the influence of various collision operators on the non-linear frequency chirping of fast-ion-driven TAEs in two different magnetic geometries. A tokamak case as well as a Wendelstein $7-X(W 7-X)$ high-mirror equilibrium have been considered. The effects of pitch-angle collisions and fast-ion drag on the non-linear dynamics of the mode were discussed separately. Pitch-angle collisions together with fast-ion drag constitute the fast-ion collision operator. A particle source was emulated using a Krook operator.

Starting with the tokamak case, we found that pitch-angle collisions lead to a reduction of the long-term saturation level and a more narrow frequency chirping compared with the collisionless case. The situation is different for the W7-X case, where the fast-ion temperature is lower while, at the same time, the fast-ion density is higher. Both effects lead to higher collision frequencies. Consequently, the non-linear dynamics of the TAE in W7-X becomes stationary for self-consistently calculated pitch-angle scattering frequencies. Contrary to the tokamak case, the long-term saturation level is enhanced in W7-X, when (self-consistently calculated) pitch-angle collisions are considered. This could be of practical relevance for the operation of the machine.

Many of the features we see in our simulations (in the tokamak as well as in the stellarator) are very similar to features observed in the one-dimensional Berk-Breizman paradigm. The drag term, for example, manifests itself in the usual way that only up-chirping frequency branches (also dubbed holes in phase space [4]) remain. The so-called clumps disappear. For some collision operators, however, differences become apparent. This concerns the influence of pitch-angle collisions on the non-linear devel- 
opment of the mode. It makes a difference whether a phase-space gradient or a density gradient in real space is considered. Furthermore, the Berk-Breizman model was found to not be applicable when a time-dependent damping rate $\gamma_{d}(t)$ is used, which is likely caused by violating the assumption of a marginal mode.

In order to simulate periodic chirping events, a particle source that restores the original distribution function needs to be present. Since the inclusion of a 'real' particle source into EUTERPE is a matter of on-going research, we use a Krook operator to emulate a particle source in the present work. The rate of reconstruction of the distribution function, $v_{\text {Krook}}$, was chosen in a way to have periodic chirping events nicely visible in proof-of-principle calculations. Experimental measurements are needed to constrain this parameter. The Krook operator is found to have the same effect in the tokamak and in the stellarator.

The issue of frequency chirping is of practical relevance for operation phases of W7-X that include NBI heating. We hope that, when experimentally measured time traces of $\delta B$ become available, we can spectroscopically determine $\gamma_{L}, \gamma_{d}$, and collision frequencies in W7-X by choosing critical input parameters of the simulations such that they fit the measurements. Such an approach has, for instance, been suggested in Ref. [27].

There could, however, be a number of problems: The Berk-Breizman model predicts $[3,25]$ that during a frequency chirp the frequency changes as $\delta \omega= \pm \alpha \gamma_{L} \sqrt{\gamma_{d} t}$. This means that, in order for the chirping to be nicely visible, both $\gamma_{L}$ and $\gamma_{d}$ should be large. However, the simulations presented in this paper indicate that the fast-ion drive in W7-X is rather small (and smaller than for the ITPA tokamak case). This leads to a narrow chirping parabola, which could be hard to resolve experimentally.

Furthermore, it was found that the collision frequencies in W7-X are high. For that reason, the periodic non-linear behaviour of $\delta B$ is suppressed. Finding parameter regimes that allow for frequency chirping could be an experimental challenge. Nevertheless, this paper shows that collisions, especially in dense W7-X plasmas with a low fast-ion temperature, significantly influence the non-linear dynamics and the saturation level. They have thus to be included in the non-linear modelling of any AE instability.

From a numerical and theoretical standpoint, this paper confirms that CKA-EUTERPE can routinely be used to perform non-linear simulations for W7-X.

Finally, we want to point out that here we considered the interaction of fast ions with a single mode. In realistic experiments, however, multiple modes - possibly close together in frequency - may be present. In that case the assumption of a fixed mode structure would need to be revisited. Work on a multi-mode version of CKA-EUTERPE is currently in progress. Thus, frequency chirping in more complex systems will be investigated in the future, once this version is operational.

\section{ACKNOWLEDGEMENT}

We would like to thank Udo von Toussaint for pointing out the benefits of modern signal processing tools. Further, we would like to acknowledge the useful comments and suggestions from the anonymous referees.

The simulations were performed on the MARCONI supercomputer (CINECA). 
This work has been carried out within the framework of the EUROfusion Consortium and has received funding from the Euratom research and training programme 20142018 under grant agreement No 633053. The views and opinions expressed herein do not necessarily reflect those of the European Commission. 


\section{References}

[1] H. L. Berk, B. N. Breizman, and Ye. Huanchun. Scenarios for the nonlinear evolution of alpha-particle-induced Alfvén wave instability. Physical Review Letters, 68:3563, 1992.

[2] H. L. Berk, B. N. Breizman, and M. Pekker. Nonlinear Dynamics of a Driven Mode near Marginal Stability. Physical Review Letters, 76:1256, 1996.

[3] H. L. Berk, B. N. Breizman, and N. V. Petviashvili. Spontaneous hole-clump pair creation in weakly unstable plasmas. Physics Letters A, 234:213, 1997.

[4] H. L. Berk, B. N. Breizman J. Candy, M. Pekker, and N. V. Petviashvili. Spontaneous holeclump pair creation. Physics of Plasmas, 6:3102, 1999.

[5] P. L. Bhatnagar, E. P. Gross, and M. Krook. A Model for Collision Processes in Gases. I. Small Amplitude Processes in Charged and Neutral One-Component Systems. Physical Review, 94:511, 1954.

[6] A. Bierwage, K. Shinohara, Y. Todo, N. Aiba, M. Ishikawa, G. Matsunaga, M. Takechi, and M. Yagi. Self-consistent long-time simulation of chirping and beating energetic particle modes in JT-60U plasmas. Nuclear Fusion, 57:016036, 2017.

[7] Boualem Boashash. Time-Frequency Signal Analysis and Processing, A Comprehensive Reference. Academic Press, 2016.

[8] Liu Chen, R. B. White, and M. N. Rosenbluth. Excitation of Internal Kink Modes by Trapped Energetic Beam Ions. Physical Review Letters, 52:1122-1125, 1984.

[9] Christoph Slaby and Ralf Kleiber and Axel Könies. Combining electromagnetic gyro-kinetic particle-in-cell simulations with collisions. Computer Physics Communications, 218:1-9, 2017.

[10] D. S. Darrow, S. J. Zweben, S. Batha, R. V. Bundy, C. E. Bush, Z. Chang, C. Z. Cheng, H. H. Duong, J. Fang, N. J. Fisch, R. Fischer, E. D. Fredrickson, G. Y. Fu, R. F. Heeter, W. W. Heidbrink, H. W. Herrmann, M. C. Herrmann, K. Hill, E. F. Jaeger, R. James, R. Majeski, S. S. Medley, M. Murakami, M. Petrov, C. K. Phillips, M. H. Redi, E. Ruskov, D. A. Spong, E. J. Strait, G. Taylor, R. B. White, J. R. Wilson, K.-L. Wong, and M. C. Zarnstorff. Alpha particle losses from Tokamak Fusion Test Reactor deuterium-tritium plasmas. Physics of Plasmas, 3:1875-1880, 1996.

[11] H. H. Duong, W. W. Heidbrink, E. J. Strait, T. W. Petrie, R. Lee, R. A. Moyer, and J. G. Watkins. Loss of energetic beam ions during TAE instabilities. Nuclear Fusion, 33:749, 1993.

[12] V. Erckmann, H.-J. Hartfuß, M. Kick, H. Renner, J. Sapper, F. Schauer, E. Speth, F. Wesner, F. Wagner, M. Wanner, A. Weller, H. Wobig, the W7-AS team, and the W7-X teams at IPP Garching, FZK Karlsruhe and IPF Stuttgart. The W7-X project: scientific basis and technical realization. In 17th IEEE/NPSS Symposium Fusion Engineering (Cat. No.97CH36131), volume 1, pages 40-48, San Diego, CA, USA, 1998. IEEE.

[13] A. Könies et al. 10th IAEA Technical Meeting on Energetic Particles in Magnetic Confinement Systems (Kloster Seeon), 2007.

[14] T. B. Fehér. Simulation of the interaction between Alfvén waves and fast particles. PhD thesis, Ernst-Moritz-Arndt-Universität Greifswald, 2013.

[15] G. Y. Fu and J. W. van Dam. Excitation of the toroidicity-induced shear Alfvén eigenmode 
by fusion alpha particles in an ignited tokamak. Physics of Fluids B, 1:1949, 1989.

[16] M. García-Muñoz, N. Hicks, R. van Voornveld, I. G. J. Classen, R. Bilato, V. Bobkov, M. Bruedgam, H.-U. Fahrbach, V. Igochine, S. Jaemsae, M. Maraschek, K. Sassenberg, and ASDEX Upgrade Team. Convective and Diffusive Energetic Particle Losses Induced by Shear Alfvén Waves in the ASDEX Upgrade Tokamak. Physical Review Letters, 104:185002, 2010.

[17] G. Grieger, W. Lotz, P. Merkel, J. Nührenberg, J. Sapper, E. Sturmberger, H. Wobig, the W7X Team, R. Burhenn, V. Erckmann, U. Gasparino, L. Giannone, H. J. Hartfuss, R. Jaenicke, G. Kühner, H. Ringler, A. Weller, F. Wagner, and the W7-AS Team. Physics optimization of stellarators. Physics of Fluids B, 4:2081, 1992.

[18] W. W. Heidbrink. Basic physics of Alfvén instabilities driven by energetic particles in toroidally confined plasmas. Physics of Plasmas, 15:055501, 2008.

[19] P. Helander and D. J. Sigmar. Collisional Transport in Magnetized Plasmas. Cambridge University Press, 2002.

[20] R. Kleiber, R. Hatzky, A. Könies, A. Mishchenko, and E. Sonnendrücker. An explicit large time step particle-in-cell scheme for nonlinear gyrokinetic simulations in the electromagnetic regime. Physics of Plasmas, 23:032501, 2016.

[21] A. Könies. paper on the CKA-EUTERPE model, in preparation.

[22] A. Könies, S. Briguglio, N. Gorelenkov, T. Fehér, M. Isaev, Ph. Lauber, A. Mishchenko, D. A. Spong, Y. Todo, W. A. Cooper, R. Hatzky, R. Kleiber, M. Borchardt, G. Vlad, A. Biancalani, A. Bottino, and ITPA EP TG. Benchmark of gyrokinetic, kinetic MHD and gyrofluid codes for the linear calculation of fast particle driven TAE dynamics. Nuclear Fusion, 58:126027, 2018.

[23] A. Könies, S. Briguglio, N. Gorelenkov, T. Fehér, M. Isaev, Ph. Lauber, A. Mishchenko, D. A. Spong, Y. Todo, W. A. Cooper, R. Hatzky, R. Kleiber, M. Borchardt, G. Vlad, and ITPA EP TG. Benchmark of gyrokinetic, kinetic MHD and gyrofluid codes for the linear calculation of fast particle driven TAE dynamics. 24th IAEA Int. Conf. on Fusion Energy (San Diego, CA), 2012. http://www-naweb.iaea.org/napc/physics/FEC/FEC2012/papers/ 437_ITRP134.pdf.

[24] V. Kornilov, R. Kleiber, R. Hatzky, L. Villard, and G. Jost. Gyrokinetic global threedimensional simulations of linear ion-temperature-gradient modes in Wendelstein 7-X. Physics of Plasmas, 11:3196, 2004.

[25] M. Lesur. Effect of collisions on energetic particle-driven chirping bursts. Physics of Plasmas, 20:055905, 2013.

[26] M. Lesur, Y. Idomura, and X. Garbet. Fully nonlinear features of the energetic beam-driven instability. Physics of Plasmas, 16:092305, 2009.

[27] M. Lesur, Y. Idomura, K. Shinohara, X. Garbet, and the JT-60 Team. Spectroscopic determination of kinetic parameters for frequency sweeping Alfvén eigenmodes. Physics of Plasmas, $17: 122311,2010$.

[28] M. K. Lilley and B. N. Breizman. Convective transport of fast particles in dissipative plasmas near an instability threshold. Nuclear Fusion, 52:094002, 2012.

[29] M. K. Lilley, B. N. Breizman, and S. E. Sharapov. Effect of dynamical friction on nonlinear energetic particle modes. Physics of Plasmas, 17:092305, 2010.

[30] M. K. Lilley and R. M. Nyqvist. Formation of Phase Space Holes and Clumps. Physical 
Review Letters, 112:155002, 2014.

[31] A. V. Melnikov, L. G. Eliseev, E. Ascasíbar, A. Cappa, F. Castejón, C. Hidalgo, T. Ido, J. A. Jiménez, A. S. Kozachek, L. I. Krupnik, M. Liniers, S. E. Lysenko, K. Nagaoka, J. L. de Pablos, A. Shimizu, S. E. Sharapov, M. V. Ufimtsev, S. Yamamoto, HIBP group, and TJ-II team. Transition from chirping to steady NBI-driven Alfvén modes caused by magnetic configuration variations in the TJ-II stellarator. Nuclear Fusion, 56:076001, 2016.

[32] A. V. Melnikov, L. G. Eliseev, F. Castejón, C. Hidalgo, P. O. Khabanov, A. S. Kozachek, L. I. Krupnik, M. Liniers, S. E. Lysenko, J. L. de Pablos, S. E. Sharapov, M. V. Ufimtsev, V. N. Zenin, HIBP Group, and TJ-II Team. Study of NBI-driven chirping mode properties and radial location by the heavy ion beam probe in the TJ-II stellarator. Nuclear Fusion, 56:112019, 2016.

[33] R. M. Nyqvist, M. K. Lilley, and B. N. Breizman. Adiabatic description of long range frequency sweeping. Nuclear Fusion, 52:094020, 2012.

[34] M. N. Rosenbluth and P. H. Rutherford. Excitation of Alfvén Waves by High-Energy Ions in a Tokamak. Physical Review Letters, 34:1428, 1975.

[35] C. Slaby, A. Könies, R. Kleiber, S. Äkäslompolo, and J. Kontula. Parametric study of fastion-driven modes in Wendelstein 7-X. Journal of Physics: Conference Series, 1125:012019, 2018.

[36] Christoph Slaby, Axel Könies, Ralf Kleiber, and José Manuel García-Regaña. Effects of collisions on the saturation dynamics of TAEs in tokamaks and stellarators. Nuclear Fusion, 58:082018, 2018.

[37] R. B. White, E. Fredrickson, D. Darrow, M. Zarnstorff, R. Wilson, S. Zweben, K. Hill, Yang Chen, and Guoyong Fu. Toroidal Alfvén eigenmode-induced ripple trapping. Physics of Plasmas, 2:2871, 1995. 